\title{
Energy dissipation in soil samples during drained triaxial shearing
}

\author{
Kevin J. Hanley ${ }^{1 *}$, Xin Huang ${ }^{2}$ and Catherine O’Sullivan ${ }^{3}$ \\ ${ }^{1}$ Institute for Infrastructure and Environment, School of Engineering, The University of Edinburgh, Edinburgh \\ EH9 3JL, United Kingdom \\ ${ }^{2}$ Department of Geotechnical Engineering, Tongji University, 1239 Siping Road, Shanghai 200092, China \\ ${ }^{3}$ Department of Civil and Environmental Engineering, Skempton Building, Imperial College London, London \\ SW7 2AZ, United Kingdom
}

Keywords: Particle-scale behaviour; Friction; Discrete-element modelling; Numerical modelling; Sands; Constitutive relations

\begin{abstract}
The discrete element method was used to simulate drained triaxial compression of large-scale, polydisperse numerical samples at a range of void ratios while tracing all relevant energy components. The frictional dissipation and boundary work are almost equal regardless of sample density. The volumetric work reaches a steady value at large strain. However, the distortional work increases continually as sample deformation continues post-critical state. There is a preferential orientation for frictional dissipation at around $45^{\circ}$ to the major principal stress direction. This matches the orientation at which there is the largest number of sliding contacts. The work equations, which are fundamental in most commonly used constitutive models, are linear when plotted against deviatoric strain. The Modified Cam Clay work equation substantially over-predicts the frictional dissipation for dense samples. An alternative, thermodynamically-consistent work equation gives a much better description of frictional dissipation and is therefore recommended to ensure accuracy in modelling.
\end{abstract}

\footnotetext{
* Corresponding author. E-mail: k.hanley@ed.ac.uk; Phone: +44 (0)13 1650 5712; Fax: +44 (0)13 16506554
} 


\section{Introduction}

The most commonly-used models of soil behaviour have been formulated based on energy considerations. The original Cam Clay model was developed by assuming that plastic work is dissipated entirely by friction (Roscoe \& Schofield, 1963; Schofield \& Wroth, 1968). Modified Cam Clay addressed some of the shortcomings of this model by revising the work equation to include volumetric dissipation (Roscoe \& Burland, 1968). Since then, many modifications of the basic Cam Clay models have been proposed (Gens \& Potts, 1988) but all retain energy-based fundamentals. This is also true of many alternative constitutive models, e.g., NorSand (Jefferies, 1993; Jefferies \& Shuttle, 2002), Severn-Trent sand (Gajo \& Muir Wood, 1999) and the model of Manzari \& Dafalias (1997).

Apart from its central role in constitutive modelling of soil, energy is key in seismic analysis and geotechnical earthquake engineering. A wide range of energy-based approaches have been proposed to predict the seismic liquefaction potential of a sand (e.g., Berrill \& Davis, 1985; Law et al., 1990; Figueroa et al., 1994; Trifunac, 1995). The damping ratio, which quantifies the energy dissipated during cyclic loading, is often computed using the areas underneath and enclosed by a hysteresis loop on a stress-strain plot (e.g., experiments of Seed et al., 1986; Vucetic \& Dobry, 1991; simulations of Sitharam \& Vinod, 2010; El Shamy \& Denissen, 2012). This method of computing energy terms is limited to distinguishing energy dissipated during the loading cycle and the maximum elastic energy stored during the cycle for the entire system.

The discrete element method (DEM), a numerical tool which originated in soil mechanics (Cundall \& Strack, 1979), is capable of giving very accurate information about energy transfer and dissipation within a particulate system. DEM simulations can capture the features of soil behaviour described by the critical state framework: researchers including Ng (2009), Yan \& Dong (2011), Guo \& Zhao (2013) and Huang et al. (2014b) have confirmed that DEM simulations can give a critical state locus, while Hanley et al. (2015) and Ciantia et al. (2015) have confirmed that DEM simulations of crushable particles can generate a normal compression line. Although DEM has been widely adopted 
by the soil mechanics community since its inception (O'Sullivan, 2014), energy is not routinely tracked in DEM simulations of quasi-static monotonic tests. This is quite surprising considering the obvious importance of energy in critical-state soil mechanics and the ease with which energy can be monitored in DEM. In comparison with experimental data analyses, the energy input and dissipated can be decomposed into more fundamental components with high temporal and spatial resolution, and thereby obtain insights into the development of critical state in sheared granular materials which cannot be obtained experimentally.

In some prior studies, energy has been considered when particle crushing is permitted. Cheng et al. (2004) and Bolton et al. (2008) quantify energy dissipation in their simulations of agglomerate crushing and use continuum Cam Clay models in their interpretation of the discrete data. Wang \& Yan (2012) and Zhou et al. (2013) also consider energy in their simulations of crushable agglomerates. In simulations where crushing is neglected, as in this paper, Zhang et al. (2013) studied the balance between sliding dissipation and rolling dissipation for 2D simulations which included a rolling resistance model. El Shamy \& Denissen (2012) computed the energy dissipated prior to liquefaction in their undrained (constant volume) cyclic loading simulations. Bi et al. (2011) and Zamani \& El Shamy (2013) also show the evolution of energy dissipation for a biaxial test and various soil-foundation-structure systems, respectively. Sun et al. (2011) used granular solid hydrodynamics in conjunction with DEM to investigate elastic energy and energy dissipation during compression of a granular material.

The research described in this paper builds upon this prior body of work with three main aims. Firstly, to quantify the evolution of the individual components of energy during shearing of large-scale polydisperse numerical samples with differing initial void ratios to critical state. Secondly, to resolve a contradiction in the literature on the existence of a preferential orientation for frictional dissipation (Wang \& Yan, 2012; Zhang et al., 2013) and propose an explanation for this behaviour. Thirdly, to extend the research of Cheng et al. (2004) and Bolton et al. (2008) by linking energy calculated from micro-scale measures to work equations of macro-scale constitutive models. The simulation data are used to assess the accuracy and applicability of both the original and Modified Cam Clay work 
equations, acknowledging theoretical flaws inherent in their formulation (Collins \& Hilder, 2002; Collins \& Muhunthan, 2003). The frictional dissipation, quantified using temporal and spatial summations of per-contact friction increments, is also compared with the dissipation predicted using a thermodynamically-consistent work equation (Collins \& Hilder, 2002). This analysis, linking particlescale discrete element simulations with continuum models, enables recommendations to be made for increasing the accuracy of constitutive models.

Frictional dissipation was the only mechanism available for energy dissipation in these simulations, i.e., rotational resistance, particle crushing and numerical damping were omitted during shearing. Both rotational resistance (Wensrich \& Katterfeld, 2012; Huang et al., 2017) and commonly used damping models are numerically convenient but not physically realistic. Crushing models used in DEM are by necessity a significant simplification of reality and affect the position of the critical state line in $e-\log \left(p^{\prime}\right)$ space (Kikumoto et al., 2010). Every parameter in the model has a clear physical basis. This simplicity is appropriate in a fundamental study of the constitutive models and their associated work equations. Note also that Schofield \& Wroth (1968) assumed that that plastic work is dissipated entirely by friction; including other terms would preclude a fundamental analysis of that assumption.

\section{DEM Simulations}

Numerical samples were created containing unbreakable spherical particles with a grading approximating that of Dunkirk sand. The ratio of maximum to minimum particle diameters is 20.6; 101,623 particles were used to ensure representative volume elements that were statistically representative were achieved. Fig. 1 shows a representative sample prior to shearing and the particle size distribution. The experimental data points on Fig. 1a were measured by the authors using a Sympatec QICPIC laser scanning apparatus (Witt et al., 2004). Periodic boundaries were used to contain these cuboidal samples. These are not physical boundaries; instead opposite faces of the cell are numerically connected so that particles that leave the cell through one face are mapped back into 
the cell at the opposite face (Cundall, 1988). A global strain field is applied uniformly across the periodic cell so that relative velocities between particles anywhere in the sample include a contribution from the applied strain field. These boundary conditions ensure a uniform deformation field (Cundall, 1988), eliminate inhomogeneities at the periphery of the sample (Huang et al., 2014a) and preclude the development of shear bands. The effective continuum normal and shear stresses for the sample are denoted as $\sigma_{x}^{\prime}, \sigma_{y}^{\prime}, \sigma_{z}^{\prime}$ and $\tau_{x y}, \tau_{x z}, \tau_{y z}$, respectively, where $x, y$ and $z$ are the reference Cartesian axes; these stresses were determined by a summation over all the contacts in the sample as outlined in, e.g., Potyondy \& Cundall (2004). The normal strains, $\varepsilon_{x}, \varepsilon_{y}, \varepsilon_{z}$, and incremental normal strains, $\delta \varepsilon_{x}, \delta \varepsilon_{y}, \delta \varepsilon_{z}$, were determined from the movements of the periodic boundaries.

The samples were prepared using the two-step process described by Hanley et al. (2014). Particles were initially placed at randomly-chosen locations within a periodic cell without overlapping existing particles. Then the cell dimensions were gradually reduced using a servo-control algorithm to create isotropic, equilibrated samples at two initial mean effective stresses: $p_{0}^{\prime}=500 \mathrm{kPa}$ or $1 \mathrm{MPa}$. Interparticle friction coefficients $(\mu)$ of $0.0,0.05$ or 0.25 were used during this sample preparation process to create six isotropic samples with a range of void ratios between 0.486 and 0.593 . This method for controlling the packing density by varying $\mu$ during sample preparation is commonly used in DEM, e.g., Thornton (2000).

Once the samples had equilibrated at the required $p_{0}^{\prime}, \mu$ was set at 0.25 for all samples based on the study of Huang et al. (2014b) and the samples were again equilibrated. Each of these six samples was then sheared to critical state in a conventional drained triaxial compression simulation by applying a normal strain rate of $\dot{\varepsilon}_{z}=1 \mathrm{~s}^{-1}$. Although this strain rate exceeds those used in laboratory testing by orders of magnitude, such rates are common in DEM simulations. These simulations are quasi-static, with inertia numbers less than $8.2 \times 10^{-4}$ throughout shearing (da Cruz et al., 2005). The servo-control algorithm maintained $\sigma_{x}^{\prime}=\sigma_{y}^{\prime}=p_{0}^{\prime}=$ constant during shearing. 
Increasing $\mu$ before shearing can cause an unrealistic, artificially stiff response at small strains because contacts are prevented from sliding at the very initial stage of loading (Bernhardt et al., 2016). However, the response quickly becomes physically reasonable upon shearing (within $0.2 \%$ shear strain for Bernhardt et al., 2016) so this initial phase does not materially affect the results at critical state.

Fig. 2 shows the critical state line (CSL) for these samples in both $e-\log \left(p^{\prime}\right)$ and $q-p^{\prime}$ spaces where $q$ and $p^{\prime}$ are the deviator and mean effective stresses, respectively, and $e$ is the void ratio. In order to obtain a broad CSL in $e-\log \left(p^{\prime}\right)$ space, data previously presented in Huang et al. (2014b) were included that consider triaxial shearing of smaller Dunkirk sand samples containing 43,906 particles $(\mu=0.25)$. This smaller sample gives a similar macro-scale response to the larger one, although slightly less smooth due to the presence of fewer particles. Fig. 2 also indicates the states at the end of isotropic compression to show their position relative to the CSL. In this paper, the samples are distinguished by their initial state parameters, $\psi_{0}$, which was defined by Been \& Jefferies (1985) as the difference between the initial $e$ and $e$ at critical state at the same $p^{\prime}$. The extent to which the material response can be considered homogeneous and hence representative depends on the size of the homogenisation or averaging volume used to determine the continuum (or average) parameters relative to the particle size. In this study, the average stress and void ratio calculated in sub-layers of thickness equal to one-eighth of the length of the periodic cell were compared; the small variation amongst these values confirmed that the periodic cell can be considered a representative element volume.

The simulations were run using 16 24-core nodes of the UK's national high-performance computing facility, ARCHER. The DEM code used was a version of the open-source, MPI-parallelised code LAMMPS (Plimpton, 1995). This code was modified by the authors to include computations of various energy and work terms, a stress-control algorithm for periodically-bounded samples and a simplified Hertz-Mindlin contact model which has been substantially revised to match the Hertz implementation described by Itasca Consulting Group (2008). In summary, for two overlapping elastic spheres with radii $r_{a}$ and $r_{b}$, the normal component of the contact force, $\boldsymbol{F}_{n}$, is given by 
equation (1) where $\alpha_{n}$ is the interparticle overlap, $G_{p}$ is the particle shear modulus, $v_{p}$ is the particle Poisson's ratio, $r_{g}=\sqrt{\frac{r_{a} r_{b}}{r_{a}+r_{b}}}$ and $\boldsymbol{n}$ is a unit vector along the line joining the sphere centres:

$$
\boldsymbol{F}_{n}=\frac{4 G_{p} r_{g}}{3\left(1-v_{p}\right)} \alpha_{n}^{\frac{3}{2}} \boldsymbol{n}
$$

The tangential or shear component of the contact force, $\boldsymbol{F}_{t}$, is calculated incrementally as

$$
\begin{aligned}
& \boldsymbol{F}_{t}^{\beta}=\boldsymbol{F}_{t}^{\beta-1}-k_{t} \delta \boldsymbol{\alpha}_{t} \\
& k_{t}=\frac{4 G_{p} r_{g}}{2-v_{p}} \sqrt{\alpha_{n}}
\end{aligned}
$$

where $\beta-1$ and $\beta$ represent consecutive time-steps, $k_{t}$ is the contact shear tangent stiffness and $\delta \boldsymbol{\alpha}_{t}$ is the increment of relative shear displacement during time-step $\beta$. A slip criterion is imposed to limit the tangential force:

$$
\left|\boldsymbol{F}_{t}^{\beta}\right| \leq \mu\left|\boldsymbol{F}_{n}\right|
$$

$G_{p}=29.17 \mathrm{GPa}$ and $v_{p}=0.2$ for these simulations: realistic parameters for a quartz sand. The other required input parameters were a particle density of $2670 \mathrm{~kg} / \mathrm{m}^{3}$ and a time-step of $3 \mathrm{~ns}$. Neither damping nor gravity was active during triaxial compression.

\section{Energy Calculations}

\section{Work terms computed using macro-scale quantities}

The data available in the DEM simulations allow work and energy terms to be calculated from both a continuum (system) perspective and a particle-scale perspective. Consider firstly the overall response for the current system where only normal strains are applied. Following Muir Wood (1990), in incremental form, the boundary work input per unit volume is

$$
\delta W=\sigma_{x}^{\prime} \delta \varepsilon_{x}+\sigma_{y}^{\prime} \delta \varepsilon_{y}+\sigma_{z}^{\prime} \delta \varepsilon_{z}
$$


This can be subdivided into an increment of distortional work per unit volume, $\delta W_{d}$, causing a change of sample shape and an increment of volumetric work per unit volume, $\delta W_{v}$, causing a change of sample volume (Muir Wood, 1990). These may be calculated as

$$
\begin{aligned}
& \delta W_{d}=q \delta \varepsilon_{q} \\
& \delta W_{v}=p^{\prime} \delta \varepsilon_{v}
\end{aligned}
$$

where $\delta \varepsilon_{q}$ and $\delta \varepsilon_{v}$ are the respective increments of triaxial shear and volumetric strain, calculated using equations (8) and (9) for a triaxial element compressed in the z-direction. $q$ and $p^{\prime}$ were calculated using equations (10) and (11). The full 3D stress tensor was used in the calculation of $q$ because of its ready availability in DEM.

$$
\begin{gathered}
\delta \varepsilon_{q}=\frac{2}{3}\left(\delta \varepsilon_{z}-0.5\left(\delta \varepsilon_{x}+\delta \varepsilon_{y}\right)\right) \\
\delta \varepsilon_{v}=\frac{1}{3}\left(\delta \varepsilon_{x}+\delta \varepsilon_{y}+\delta \varepsilon_{z}\right) \\
q=\sqrt{\frac{1}{2}\left(\left(\sigma_{x}^{\prime}-\sigma_{y}^{\prime}\right)^{2}+\left(\sigma_{x}^{\prime}-\sigma_{z}^{\prime}\right)^{2}+\left(\sigma_{y}^{\prime}-\sigma_{z}^{\prime}\right)^{2}\right)+3\left(\tau_{x y}^{2}+\tau_{x z}^{2}+\tau_{y z}^{2}\right)} \\
p^{\prime}=\frac{1}{3}\left(\sigma_{x}^{\prime}+\sigma_{y}^{\prime}+\sigma_{z}^{\prime}\right)
\end{gathered}
$$

Equations (5)-(7) are all increments per unit volume. In each time-step, $\beta$, these increments were multiplied by the current sample volume, $V^{\beta}$, and added to the corresponding accumulated boundary, distortional or volumetric work terms:

$$
\begin{aligned}
& W^{\beta}=W^{\beta-1}+\delta W^{\beta} V^{\beta} \\
& W_{d}^{\beta}=W_{d}^{\beta-1}+\delta W_{d}^{\beta} V^{\beta} \\
& W_{v}^{\beta}=W_{v}^{\beta-1}+\delta W_{v}^{\beta} V^{\beta}
\end{aligned}
$$




\section{Work equations for constitutive models}

The original Cam Clay (Roscoe \& Schofield, 1963; Schofield \& Wroth, 1968) and Modified Cam Clay (Roscoe \& Burland, 1968) work equations are given in Muir Wood (1990). On a per-unitvolume basis, the total input energy increment given by equation (5) is the sum of equations (6) and (7):

$$
\delta W=\delta W_{d}+\delta W_{v}
$$

The elastic portion of this total increment is recoverable whereas the plastic portion, $\delta W^{p}$, is available for dissipation. $\delta W^{p}$ is calculated as

$$
\delta W^{p}=\delta W_{d}^{p}+\delta W_{v}^{p}=q \delta \varepsilon_{q}^{p}+p^{\prime} \delta \varepsilon_{v}^{p}
$$

Equation (16) is analogous to equation (15) except plastic strain increments are used as indicated by the superscript ' $p$ ', rather than total strain increments. In developing the original Cam Clay work equation, Schofield \& Wroth (1968) assumed that $\delta W^{p}$ is dissipated entirely by friction and used equation (17) from Taylor (1948) which is based on shear box test results:

$$
\delta W^{p}=M p^{\prime} \delta \varepsilon_{q}^{p}
$$

where $M$ is the value of the stress ratio, $\eta=\frac{q}{p^{\prime}}$, at critical state. For unbreakable particles, the extended work equation proposed by McDowell \& Bolton (1998) which includes surface energy has the same form as equation (17). Modified Cam Clay has a work equation which includes $\delta \varepsilon_{q}^{p}, \delta \varepsilon_{v}^{p}$ and $M$ :

$$
\delta W^{p}=p^{\prime} \sqrt{\left(M \delta \varepsilon_{q}^{p}\right)^{2}+\left(\delta \varepsilon_{v}^{p}\right)^{2}}
$$

In the DEM simulations presented here, at each time-step, $\beta$, these work increments per unit volume were multiplied by the current overall sample volume $V^{\beta}$ and added to the corresponding accumulated work terms as shown in equation (12). This approach is valid because the simulations are triaxial 
rather than true triaxial. In order to compute these work equations, the total strain increments were divided into elastic and plastic increments. It was assumed that

$$
\delta \varepsilon_{q}=\delta \varepsilon_{q}^{e}+\delta \varepsilon_{q}^{p}
$$

and

$$
\delta \varepsilon_{v}=\delta \varepsilon_{v}^{e}+\delta \varepsilon_{v}^{p}
$$

where $\delta \varepsilon_{q}^{e}$ and $\delta \varepsilon_{v}^{e}$ represent the elastic components of the shear and volumetric strain increments, respectively. This decomposition is applicable to volumetric strain increments (e.g., Muir Wood, 1990; Einav, 2007; Kamai \& Boulanger, 2012) and also shear strain increments if the work conjugate shear strain measure is linear (Jefferies \& Been, 2006), as in this case, e.g., equation (8). The elastic strain increments were calculated as follows (Muir Wood, 1990):

$$
\begin{aligned}
& \delta \varepsilon_{v}^{e}=\frac{\delta p^{\prime}}{K}=\frac{\kappa \delta p^{\prime}}{(1+e) p^{\prime}} \\
& \delta \varepsilon_{q}^{e}=\frac{\delta q}{3 G}
\end{aligned}
$$

where $(1+e)$ is the specific volume, $K$ and $G$ are the respective bulk and shear moduli of the sample, and $\kappa$ is the gradient of the swelling line, i.e., the negative slope of the unloading-reloading line in $(1+e): \ln \left(p^{\prime}\right)$ space. $K$ changes during shearing due to changes in $e$ and (primarily) in $p^{\prime} . G$ is assumed to be constant while the Poisson's ratio of the sample $v$ is allowed to vary. This is the preferred option for an energy-based analysis, even though a constant $G$ does not match experimental observations. While it is usually acceptable to make the opposite assumption, i.e., to fix $v$ and allow $G$ to vary, the resulting model does not conserve energy during closed stress cycles (Zytynski et al., 1978; Gens \& Potts, 1988). Once the elastic and total increments of strain are known, the plastic increments are calculated from equations (19) and (20).

Three parameters are required in the DEM code to calculate the work equations: $M, G$ and $\kappa . M$ was set at 0.687: the slope of the best-fit critical state line in $q-p^{\prime}$ space based on several preliminary 
drained triaxial simulations. Small-strain simulations, limited to a shear strain, $\varepsilon_{q}$, of around $5 \times 10^{-7}$, were used to find $G$ and $\kappa$ for each sample. $G$ was taken to be one-third of the slope of a linear regression fitted to a $q-\varepsilon_{q}$ plot. Once $G$ is known, $\kappa$ may be found from a $\varepsilon_{v}-\varepsilon_{q}$ plot, the slope of which is $\frac{G}{K}=\frac{\kappa G}{(1+e) p^{\prime}}$ (Muir Wood, 1990). The validity of this method for finding $\kappa$ was confirmed by comparing with the results to one-dimensional load-unload oedometer tests; these tests were restricted to a maximum strain of 0.0003 and so the hysteresis was negligible. The small-strain elastic parameters are given in Table 1.

Table 1. Initial void ratios, mean effective stresses, state parameters and elastic parameters of the samples

\begin{tabular}{|c|c|c|c|c|c|}
\hline $\begin{array}{c}\mu \text { during } \\
\text { isotropic } \\
\text { compression }\end{array}$ & $\begin{array}{c}\text { Initial mean } \\
\text { effective stresses, } \\
p_{0}^{\prime}(\mathrm{kPa})\end{array}$ & $\begin{array}{c}\text { Initial state } \\
\text { parameter, } \\
\psi_{0}\end{array}$ & $\begin{array}{c}\text { Initial } \\
\text { void ratio, } \\
e_{0}\end{array}$ & $\begin{array}{c}\text { Shear } \\
\text { modulus, } G \\
(\mathrm{MPa})\end{array}$ & $\begin{array}{c}\text { Gradient of } \\
\text { swelling line, } \\
\kappa\end{array}$ \\
\hline 0.0 & 500 & -0.0923 & 0.489 & 398 & 0.00266 \\
\hline 0.05 & 500 & -0.0457 & 0.536 & 340 & 0.00320 \\
\hline 0.25 & 500 & 0.0117 & 0.593 & 154 & 0.00721 \\
\hline 0.0 & 1000 & -0.0948 & 0.486 & 511 & 0.00424 \\
\hline 0.05 & 1000 & -0.0475 & 0.533 & 442 & 0.00500 \\
\hline 0.25 & 1000 & 0.0098 & 0.591 & 212 & 0.01075 \\
\hline
\end{tabular}

In addition to equations (17) and (18), two other forms of these work equations were computed. The Cam Clay work equation was computed assuming irreversible shear strains, i.e., $\delta \varepsilon_{q} \approx \delta \varepsilon_{q}^{p}$ and $\delta W^{p}=M p^{\prime} \delta \varepsilon_{q}$. Building on the work of Houlsby (1981), Collins and his co-workers highlighted theoretical inconsistencies in the development of the Modified Cam Clay work equation (Collins \& Hilder, 2002; Collins \& Muhunthan, 2003). They proposed the thermodynamically consistent 
statement of the Modified Cam Clay work equation given by equations (23)-(25) where $p_{c}^{\prime}$ is the mean normal consolidation pressure:

$$
\begin{aligned}
& d \Psi_{2}=\frac{p_{c}^{\prime}}{2} \delta \varepsilon_{v}^{p} \\
& \delta \Phi=\frac{p_{c}^{\prime}}{2} \sqrt{\left(M \delta \varepsilon_{q}^{p}\right)^{2}+\left(\delta \varepsilon_{v}^{p}\right)^{2}} \\
& \delta W^{p}=d \Psi_{2}+\delta \Phi
\end{aligned}
$$

$d \Psi_{2}$, which may be positive or negative, represents the recoverable contribution to the plastic work from the free energy caused by micro-scale rearrangements (Collins \& Hilder, 2002). $\delta \Phi$ represents the dissipative part of the plastic work and is always positive. $\delta \Phi$ is closely related to the conventional Modified Cam Clay work equation given by equation (18): the equations differ by a factor of $\frac{M^{2}+\eta^{2}}{2 M^{2}}$, implying they become equal at critical state when $\eta=M$. The sum of $d \Psi_{2}$ and $\delta \Phi$ gives the total increment of the plastic work per unit volume. These terms were computed within the DEM code in the same manner as the conventional work equations for original and Modified Cam Clay. $p_{c}^{\prime}$ was calculated using equation (26), assuming an elliptical yield locus (Muir Wood, 1990):

$$
p_{c}^{\prime}=p^{\prime} \frac{M^{2}+\eta^{2}}{M^{2}}
$$

\section{Energy terms computed from micro-mechanics}

The particle-scale energy terms were also traced. The translational and rotational kinetic energies are respectively calculated as

$$
\begin{aligned}
& E_{k t}=\frac{1}{2} \sum_{i=1}^{N_{p}} m_{i} v_{i}^{2} \\
& E_{k r}=\frac{1}{2} \sum_{i=1}^{N_{p}} I_{i} \omega_{i}^{2}
\end{aligned}
$$


where $N_{p}$ is the total number of particles, $m_{i}, v_{i}$ and $\omega_{i}$ are the mass, translational speed and rotational speed, respectively, of particle $i$, and $I_{i}=0.4 m_{i} r_{i}^{2}$ is the moment of inertia of a spherical particle $i$.

Energy is dissipated by frictional sliding whenever equation (4) is invoked. The friction dissipated at contact $j$ during time-step $\beta$ is

$$
\delta E_{f_{-} j}^{\beta}=\left\{\begin{array}{r}
\frac{\left|\boldsymbol{F}_{t}^{\beta-1}+\boldsymbol{F}_{t}^{\beta}\right|}{2} \frac{\left|\boldsymbol{F}_{t_{-} o}^{\beta}-\boldsymbol{F}_{t}^{\beta}\right|}{k_{t}},\left|\boldsymbol{F}_{t_{-} o}^{\beta}\right|>\mu\left|\boldsymbol{F}_{n}\right| \\
0,\left|\boldsymbol{F}_{t_{-} o}^{\beta}\right| \leq \mu\left|\boldsymbol{F}_{n}\right|
\end{array}\right.
$$

using $\boldsymbol{F}_{t_{-} o}^{\beta}$ to represent the tangential force computed using equation (2) before applying the slip criterion (Itasca Consulting Group, 2008). The non-zero case for $\delta E_{f_{-} j}^{\beta}$ is a product of two terms. The first is the magnitude of the average tangential force at contact $j$ at the beginning and end of time-step $\beta$. The second term is the magnitude of the slip displacement: the change in magnitude of the tangential force as a result of applying equation (4) divided by the shear tangent stiffness. For each contact, the frictional dissipation is accumulated:

$$
E_{f_{-} j}^{\beta}=E_{f_{-} j}^{\beta-1}+\delta E_{f_{-} j}^{\beta}
$$

The normal component of strain energy is found as the area underneath a graph of $\left|\boldsymbol{F}_{n}\right|$ against $\alpha_{n}$. This is given by equation (31) for a single Hertzian contact, $j$, by substituting equation (1):

$$
E_{S n_{-} j}=\int_{0}^{\alpha_{n}}\left|\boldsymbol{F}_{n}\right| d \alpha_{n}=\frac{4 G_{p} r_{g}}{3\left(1-v_{p}\right)}|\boldsymbol{n}| \int_{0}^{\alpha_{n}} \alpha_{n}^{\frac{3}{2}} d \alpha_{n}=\frac{2}{5}\left|\boldsymbol{F}_{n}\right| \alpha_{n}
$$

Unlike the normal component of strain energy, the tangential component requires incremental calculation. This calculation is done after the magnitude of the tangential force, $\left|\boldsymbol{F}_{t}^{\beta}\right|$, has been rescaled to $\mu\left|\boldsymbol{F}_{n}\right|$ if necessary. At any contact, $j$,

$$
E_{s t_{-} j}^{\beta}=E_{s t_{-} j}^{\beta-1}+\delta E_{s t_{-} j}^{\beta}
$$




$$
\delta E_{s t_{-} j}^{\beta}=\frac{\left|\boldsymbol{F}_{t}^{\beta-1}+\boldsymbol{F}_{t}^{\beta}\right|}{2} \frac{\left|\boldsymbol{F}_{t}^{\beta}-\boldsymbol{F}_{t}^{\beta-1}\right|}{k_{t}}
$$

$\delta E_{s t_{-} j}^{\beta}$ has a similar form to $\delta E_{f_{-} j}^{\beta}$, being the product of two terms: the magnitude of the average tangential force at contact $j$ at the beginning and end of time-step $\beta$, and the magnitude of the incremental displacement. This incremental displacement differs from $\delta \boldsymbol{\alpha}_{t}$ if contact sliding occurs.

The total normal and tangential components of strain energy and the frictional dissipation by timestep $\beta$ are found by summation over all $N_{c}$ contacts:

$$
\begin{aligned}
& E_{s n}=\sum_{j=i}^{N_{c}} E_{s n_{-} j} \\
& E_{s t}^{\beta}=E_{s t}^{\beta-1}+\sum_{j=i}^{N_{c}} \delta E_{s t_{-} j}^{\beta} \\
& E_{f}^{\beta}=E_{f}^{\beta-1}+\sum_{j=i}^{N_{c}} \delta E_{f_{-} j}^{\beta}
\end{aligned}
$$

Within the simulations, the energy terms are stored for each contact and also for the whole system. As the tangential components of strain energy are calculated incrementally, a small error inevitably accrues over a large number of time-steps. When two particles lose contact, there will be a tiny, residual value of $E_{s t_{-} j}$ which may be positive or negative. This residual strain energy is transferred to the accumulated frictional dissipation, i.e., $E_{s t_{-} j}$ is subtracted from $E_{s t}^{\beta}$ and added to $E_{f}^{\beta}$. This small correction ensures that the accumulated errors in the strain energy are minimised.

All work terms are calculated from discrete or incremental data, rather than being obtained by continuous integration; consequently they are only accurate within a tolerance. The large number of particles and small increments used here served to minimise this discretisation error.

\section{Results and Discussion}




\section{$\underline{\text { Stress-strain behaviour and scalar measures of fabric }}$}

The evolution of stress ratio, $\eta$, and $\varepsilon_{v}$ with axial strain are shown in Fig. 3. Each sample attains a clear critical state before $30 \%$ axial strain. As expected, the samples with negative initial state parameters dilate throughout shearing (Been \& Jefferies, 1985). The loosest samples initially contract prior to dilating and overall have a contractive response.

The coordination number, $Z$, is a scalar measure of the fabric, i.e., the internal topology of the

granular material. It is defined as the average number of contacts per particle: $Z=2 \frac{N_{c}}{N_{p}}$ where $N_{p}$ and $N_{c}$ are the numbers of particles and of interparticle contacts, respectively. The mechanical coordination number, $Z_{m}$, is a variant which was defined by Thornton (2000) to be the average number of contacts per particle excluding those with fewer than two contacts. $Z, Z_{m}$ and the fraction of contacts which are sliding are all plotted in Fig. $4 . Z_{m}$ stabilises at around 5 before $5 \%$ axial strain is attained and remains almost constant thereafter. $Z$ shows more variability before critical state at which stable values of 3.6-3.85 are attained. These results are in qualitative agreement with Thornton (2000). Although samples with similar $\psi_{0}$ show similar macro-scale behaviour (Fig. 3), the (mechanical) coordination number for samples with similar $\psi_{0}$ is sensitive to $p_{0}^{\prime}$. The sliding fractions of around $20 \%$ at critical state in Fig. $4 \mathrm{c}$ are similar to those for simulations of Toyoura sand using an interparticle friction coefficient of 0.25 (Huang et al., 2014b).

Fig. 3 and Fig. 4 show that the samples at a similar $\psi_{0}$ show similar trends; this remains true for other measures considered in this paper. Therefore, all subsequent figures contain data only for those three simulations with $p_{0}^{\prime}=500 \mathrm{kPa}\left(\psi_{0}=-0.0923,-0.0457,0.0117\right)$.

\section{Evolution of energy during shearing}

To confirm that all sources of energy dissipation were correctly identified, and that the expressions for each energy component are appropriate and have been correctly implemented in the DEM code, the error in the energy balance at any time-step $\beta, \Delta E$, was calculated as 


$$
\Delta E=E_{s n}^{0}+E_{s t}^{0}+E_{k t}^{0}+E_{k r}^{0}+W^{\beta}-E_{f}^{\beta}-E_{s n}^{\beta}-E_{s t}^{\beta}-E_{k t}^{\beta}-E_{k r}^{\beta}
$$

In this expression, terms 1-4 represent the strain and kinetic energy at the start of shearing, i.e., at time-step 0 . Terms 5-10 respectively indicate total boundary work input between time-steps 0 and $\beta$ (equation (12)), friction dissipated between time-steps 0 and $\beta$ (equation (36)), normal (equation (34)) and tangential (equation (35)) components of strain energy at time-step $\beta$, and translational (equation (27)) and rotational (equation (28)) kinetic energies at time-step $\beta$. This error was divided by boundary work and expressed as a percentage in Fig. 5. The percentage error is completely negligible, smaller than $1 \times 10^{-5} \%$ at critical state; confirming that the system achieves an energy balance and that there is no spurious generation of energy as might occur in the case of numerical instability. Because the boundary work is zero at the start of shearing, and $<6 \mu \mathrm{J}$ at a strain of $0.001 \%$, the percentage error on Fig. 5 appears large during the initial time-steps. However, the magnitude of the error is tiny: $<1.2 \mathrm{~nJ}$ at $1 \%$ axial strain, increasing to a maximum of $19 \mathrm{~nJ}$ at the end of shearing.

Fig. 6 shows the evolution of the key energy terms with axial strain: boundary work, frictional dissipation and strain energy divided into normal and tangential/shear components. The translational and rotational kinetic energy terms are negligible: $<5 \times 10^{-7} \mathrm{~J}$ at all strains. Boundary work and frictional dissipation are almost equal (Fig. 6a), supporting the assumption made by Schofield \& Wroth (1968) that $\delta W^{p}$ is dissipated entirely by friction. Prior research by Zamani \& El Shamy (2013) supports the finding that interparticle frictional sliding is the main source of energy dissipation. Frictional dissipation is insensitive to $\psi_{0}$ and continues indefinitely, even after the attainment of critical state. Conversely, the normal (Fig. 6b) and shear (Fig. 6c) components of strain energy become constant at large strain. The strain energy terms reach the same values irrespective of $\psi_{0}$ at a fixed $p_{0}^{\prime}$. Changing $p_{0}^{\prime}$ from $500 \mathrm{kPa}$ to $1 \mathrm{MPa}$ causes the critical state strain energy values to increase. The trends in strain energy closely match those in stress ratio shown in Fig. 3a: the strain energy terms for the densest sample rise sharply to a peak before decreasing to critical state, whereas the pronounced peak is absent for the loose sample. At critical state, the ratio of shear to normal strain 
energy components converges to a value of around 0.0275 ; however, this ratio is likely to depend on factors such as the grading and $\mu$.

The total boundary work may be divided into distortional and volumetric work terms which are compared in Fig. 7. The distortional work increases continually because the increment of triaxial shear strain (equation (8)) is strictly positive, $q>0$ and $\delta W_{d}=q \delta \varepsilon_{q}$. Conversely, the increment of volumetric work per unit volume is zero at critical state because the volumetric strain becomes constant (Fig. 3b). The trends for volumetric work parallel those for volumetric strain: dense samples dilate, i.e., $\delta \varepsilon_{v}<0$, so $\delta W_{v}<0$ from equation (7) as $p^{\prime}>0$. However, as a loose sample contracts, $\delta \varepsilon_{v}$ $>0$ and $\delta W_{v}>0$. Fig. $3 \mathrm{~b}$ shows that both samples with negative $\psi_{0}$ have a dilative response and these samples have $W_{v}<0$ at critical state. The loosest sample is overall contractive and its volumetric work is positive at critical state. The sum of the distortional and volumetric work terms is compared with the total boundary work in Fig. 7a. The absolute difference between the two is $<3 \times 10^{-5} \mathrm{~J}$ for all simulations at all strains.

\section{Orientation of preferential frictional dissipation}

Fig. 6a shows the evolution of frictional dissipation during shearing. In Fig. 8, the total frictional dissipation at critical state is considered based on spatial orientation of the branch vectors associated with each contact using rose diagrams. In each Cartesian plane $(x-y, x-z$ and $y-z), 18$ angular increments of $10^{\circ}$ were considered between $0^{\circ}$ and $180^{\circ}$. The length of each segment or bin in Fig. 8 is proportional to the total frictional dissipation at critical state, where critical state is taken as the end of each simulation, for contacts with branch vectors oriented within that increment. The colour of each segment indicates the frictional dissipation which has occurred.

For the horizontal $x-y$ planes, frictional dissipation is isotropic (Fig. 8a). However, there is a clear preferential orientation for the $x-z$ and $y-z$ planes. More frictional dissipation occurs for contacts oriented at around $45^{\circ}$ than for any other orientation. Those contacts which are oriented horizontally are responsible for least energy dissipation. Zhang et al. (2013) previously showed the development 
of anisotropy in sliding dissipation during shearing using 2D simulations with rolling resistance. Interestingly, Wang \& Yan (2012) reported that "the distribution of accumulated friction dissipation at large strains usually lacks a strongly preferred orientation". The inclusion of particle crushing or the development of large shear bands in the simulations of Wang \& Yan (2012) might explain this apparent contradiction.

Although Fig. 8 is independent of $\psi_{0}$, there is considerable variation in the equivalent rose diagrams prior to critical state. It is impractical to present sets of rose diagrams at a series of different axial strains. Instead, the coefficient of variation $\left(c_{v}\right)$, defined as the standard deviation divided by the mean, was computed for each set of $36 x-z$ and $y-z$ bins at each axial strain. Rose diagrams such as those in Fig. 8 a have $c_{v} \approx 0$. As the bins become increasingly dissimilar, $c_{v}$ increases. The variation of $c_{v}$ with axial strain is shown in Fig. 9. At critical state, all samples have a similar $c_{v}$ of around 0.2. $c_{v}$ increases most rapidly for the densest, most dilative sample.

The reason for the existence of this preferential orientation for frictional dissipation is summarised in Fig. 10 and Fig. 11. The data are shown for only one simulation with $\psi_{0}=-0.0457$ as the other simulations show similar behaviour. Fig. 10 shows that the number of interparticle contacts and the mean normal or shear forces at a contact are all highest in the direction of the major principal stress. Fewest contacts are oriented horizontally and these contacts carry lower forces, on average, than contacts at other orientations. However, the average ratio of tangential to normal force and the sliding contact fraction show the opposite trend in Fig. 11: both measures are highest for horizontallyoriented contacts and lowest for vertically-oriented contacts. This means that the horizontally-oriented contacts are most likely to slide but are relatively few in number, while the vertically-oriented contacts are most numerous but are least likely to slide. Thus, it makes sense that the largest number of sliding contacts, i.e., the number of contacts for which $\left|\boldsymbol{F}_{t, o}^{\beta}\right|>\mu\left|\boldsymbol{F}_{n}\right|$ in equation (29), would be found at some intermediate orientation between horizontal and vertical. The central column of Fig. 11 shows this to be the case. Because sliding is a precondition for frictional dissipation, it thus follows that most frictional dissipation will occur at a similar orientation which explains the results presented 
in Fig. 8. It is also noted that the preferred orientation for frictional dissipation is close to the orientation of the maximum shear stress: $45^{\circ}$.

Satake (1982) proposed the second-order fabric tensor, $\Phi_{p q}$, as a useful measure of fabric anisotropy:

$$
\Phi_{p q}=\sum_{j=1}^{N_{c}} \boldsymbol{n}_{p}^{j} \boldsymbol{n}_{q}^{j}
$$

where $\boldsymbol{n}_{p}^{j}$ is the unit contact normal for contact $j$. The deviatoric fabric is the difference between the maximum and minimum eigenvalues of the $\Phi_{p q}$ tensor. Fig. 12 shows the variation of deviatoric fabric with axial strain, along with the sliding deviatoric fabric: a variant computed only with the subset of sliding contacts. The deviatoric fabric matches previous observations, e.g., Huang et al. (2014b). The trends are similar to those for stress ratio (Fig. 3a): peak anisotropy increases with sample density. The anisotropy is much lower when restricted to sliding contacts only, with the deviatoric fabric being approximately halved at critical state. This might be inferred from the rose diagrams in Fig. 10 and Fig. 11: the leftmost column of Fig. 10 shows a strong preferential alignment for all contacts in the direction of the major principal stress whereas the middle column of Fig. 11 shows a less pronounced preferential orientation.

\section{Dilatancy and rate of energy dissipation}

In order to compute plastic dilatancy and the work equations presented in the following subsection, the total strain was divided into elastic and plastic components according to equations (19)-(22). Very small strains are elastic. However, the elastic shear strains quickly become insignificant as shearing proceeds, showing that the common assumption of negligible elastic deviator strains, e.g., Cheng et al. (2004), is reasonable. The elastic proportion of volumetric strain is far more significant; this proportion becomes constant at critical state as does the total volumetric strain. Fig. 13 shows that there is a systematic trend in shear and volumetric strains with $\psi_{0}$ : at critical state, elastic strains are a lesser proportion of the total strains for denser samples. 
The dilation rate was computed as $D^{p}=\frac{\delta \varepsilon_{v}^{p}}{\delta \varepsilon_{q}^{p}}$ based on these approximated plastic strain increments. A moving average filter was used to smooth the incremental strain data before plotting the (plastic) dilatancy in Fig. 14a. The Cam Clay flow rule, which approximates $D^{p}$ as $M-\eta$, is also shown in Fig. 14a as dashed lines. Here $\eta$ is the exact value computed as $\frac{q}{p^{\prime}}$ using the DEM data. The error in this approximation increases with sample density: the dilatancy of the loose sample is very well described by $M-\eta$ whereas the peak $D^{p}$ is poorly captured for the samples with $\psi_{0}<0$.

Fig. 14b shows the rate of change of frictional dissipation with respect to axial strain, using the same smoothing approach. The three simulations give very similar results except at small strain. This disparity at small strain is an artefact of the method used for sample preparation. Both samples with $\psi_{0}<0$ were prepared by isotropic compression using $\mu<0.25$. The interparticle friction coefficient was then increased to 0.25 before shearing. As discussed by Bernhardt et al. (2016), when this approach is used contacts are far from being in a sliding state at the start of shearing. When the same $\mu$ value was used during sample preparation and shearing to create the loose sample, frictional dissipation could occur from the first time-step. Therefore, there is an initial jump for the loose sample in Fig. 14b.

Summing $D^{p}$ and $\eta$ gives the dimensionless plastic energy dissipation rate (Jefferies \& Been, 2006): the normalised rate of work per unit of plastic distortion. This is plotted in Fig. 14c. Consider the loose sample with $\psi_{0}=0.0117$. The magnitude of the volumetric and distortional work terms is low compared to the dense samples (Fig. 7) as is the magnitude of the irrecoverable volumetric strain (Fig. 3b). However, all three samples have similar boundary work input (Fig. 7a). Therefore, the rate of work input per unit of distortion is necessarily largest for the loose sample prior to critical state.

\section{Work equations used in constitutive models}

The various forms of the work equations are compared with the frictional dissipation, which is almost equal to the input boundary work (Fig. 6a), in Fig. 15. All become linear when plotted against 
deviatoric strain, $\varepsilon_{q}$, where $\varepsilon_{q}=\frac{2}{3}\left(\varepsilon_{z}-\frac{\varepsilon_{x}+\varepsilon_{y}}{2}\right)$ for these triaxial tests. The original Cam Clay work equation is almost unchanged if irreversible shear strains are assumed, i.e., if plastic strains are substituted by total strains. For the loosest sample shown in Fig. 15c, the work equations are all very similar and $d \Psi_{2} \approx 0$. The differences are magnified as the samples become denser, as shown clearly in Fig. 16 in which the differences between the work equations and frictional dissipation are presented. For the densest sample (Fig. 16a), the Modified Cam Clay work equation substantially over-predicts the actual dissipation which occurs. The same occurs for original Cam Clay, but the difference is smaller. For both denser samples, $d \Psi_{2}<0$ and $\delta \Phi>E_{f}^{\beta}$ (the frictional dissipation). The thermodynamically-consistent work equation given as equation (25) matches the actual frictional dissipation almost perfectly.

Theoretical flaws in the commonly-used work equations based on Cam Clay have been known for a long time (Houlsby, 1981). This DEM analysis, based on a simple model of spherical particles without numerical damping, rotational resistance or grain crushing, quantitatively shows a significant inaccuracy for simulations of dense sands. These findings differ from those of Cheng et al. (2004) who, using a different analytical approach, concluded that Modified Cam Clay describes the behaviour of crushable sands well apart from the lack of normality.

\section{Conclusions}

This paper was introduced with three main aims: to investigate the nature of energy dissipation during triaxial compression, to explore the existence of a preferential orientation for frictional dissipation and to assess commonly used constitutive models from the perspective of energy. The conclusions presented below correspond to these aims; these conclusions are based on an analysis of energy in a set of large-scale, highly polydisperse numerical samples which were sheared triaxially to critical state. The frictional dissipation and boundary work are almost equal regardless of sample density. Frictional dissipation is insensitive to $\psi_{0}$ throughout shearing, while the normal and shear 
components of strain energy attain constant values at critical state which are independent of $\psi_{0}$. When the total boundary work was divided into distortional and volumetric work terms, it was found that the distortional work increases continually, even after the attainment of critical state, but the volumetric work reaches a steady value at large axial strain.

The second aim of this paper was met by conclusively demonstrating the existence of a preferential orientation for frictional dissipation using rose diagrams. More frictional dissipation occurs for contacts oriented at around $45^{\circ}$ to the major principal stress direction than for any other orientation, coinciding with the plane of maximum shear stress. This matches the orientation at which there is the largest number of sliding contacts. The underlying reason for this preferential orientation is the interplay between the number of interparticle contacts and the average ratio of tangential to normal force: contacts which are most numerous, aligned with the major principal stress, have the lowest average ratio of tangential to normal force and hence are least likely to slide. The anisotropy is much lower for the subset of sliding contacts than all contacts based on both deviatoric fabric calculations and rose diagrams.

In the fulfilment of the third aim, it was found that elastic shear strains are negligible but the elastic proportion of volumetric strain is much more substantial. As sample density decreases, elastic strains become an increasingly significant proportion of the total strains at critical state. All computed work equations are linear when plotted against deviatoric strain. For loose samples, all work equations give a good description of the actual frictional dissipation which occurs during shearing. However, disparities increase with increasing sample density. The Modified Cam Clay work equation substantially over-predicts the frictional dissipation which occurs for the densest sample. The equivalent thermodynamically-consistent work equation (Collins \& Hilder, 2002) matches the actual frictional dissipation almost perfectly. Based on the analysis presented in this paper, the adoption of a thermodynamically-consistent formulation is advised to ensure accuracy in modelling. This is a good example of how discrete element simulations have practical value as a tool to inform the development and application of continuum constitutive models. 


\section{Acknowledgements}

This work used the ARCHER UK National Supercomputing Service (http://www.archer.ac.uk). Part of this research was undertaken during a collaborative research visit funded by an EC FP7-PEOPLE2011-IRSES project: Marie Curie Action 294976, 'Geohazards and Geomechanics'. The second author would also like to acknowledge the financial support of National Science Foundation of China (No. 51509186).

\section{Notation}

$c_{v} \quad$ Coefficient of variation, i.e., standard deviation divided by the mean

$d \Psi_{2} \quad$ Recoverable contribution to the plastic work from the free energy

$D^{p} \quad$ Dilatancy computed using plastic strains

$e, e_{0} \quad$ Void ratio during shearing and after isotropic compression

$E_{f}^{\beta-1}, E_{f}^{\beta} \quad$ Total frictional dissipation which has occurred at time-steps $\beta-1$ and $\beta$

$E_{f_{-} j}^{\beta-1}, E_{f_{-} j}^{\beta} \quad$ Energy dissipated at contact $j$ at time-steps $\beta-1$ and $\beta$

$E_{k r}, E_{k r}^{0}, E_{k r}^{\beta} \quad$ Rotational kinetic energy, energy at the start of shearing and at time-step $\beta$

$E_{k t}, E_{k t}^{0}, E_{k t}^{\beta} \quad$ Translational kinetic energy, energy at the start of shearing and at time-step $\beta$

$E_{s n}, E_{s n}^{0}, E_{s n}^{\beta} \quad$ Total normal strain energy, energy at the start of shearing and at time-step $\beta$

$E_{S n_{-} j} \quad$ Normal component of strain energy at contact $j$

$E_{s t}^{\beta-1}, E_{s t}^{\beta} \quad$ Total tangential strain energy at time-steps $\beta-1$ and $\beta$ 
$E_{s t_{-} j}^{\beta-1}, E_{s t_{-} j}^{\beta}$

$\boldsymbol{F}_{n}$

$\boldsymbol{F}_{t}$

$\boldsymbol{F}_{t \_o}^{\beta}$

G

$G_{p}$

$i$

$I_{i}$

j

K

$k_{t}$

M

$m_{i}$

$\boldsymbol{n}$

$$
\boldsymbol{n}_{p}^{k}
$$

$N_{c}$

$N_{p}$

$p^{\prime}$

$p_{0}^{\prime}$
Tangential component of strain energy at contact $j$ at time-steps $\beta-1$ and $\beta$

Normal component of the contact force

Tangential/shear component of the contact force

Tangential force before applying Coulomb slip criterion

Sample shear modulus

Particle shear modulus

Identifier of a single particle in the system

Moment of inertia of particle $i$

Identifier of a single Hertzian contact in the system

Bulk modulus

Contact shear tangent stiffness

Critical state value of the stress ratio, $\eta$

Mass of particle $i$

Unit branch vector

Unit contact normal for contact $j$

Total number of interparticle contacts in the simulation

Total number of particles in the simulation

Mean effective stress

Initial mean effective stress after isotropic compression 
$r_{a}, r_{b}$

$r_{g} \quad \sqrt{\frac{r_{a} r_{b}}{r_{a}+r_{b}}}$

$V^{\beta}$

$v_{i}$

$W^{\beta-1}, W^{\beta}$

$W_{d}^{\beta-1}, W_{d}^{\beta}$

$W_{v}^{\beta-1}, W_{v}^{\beta}$

$x, y, z$

Z

$Z_{m}$

$\alpha_{n}$

$\beta$

$\delta E_{f_{-} j}^{\beta}$

$\delta E_{s t_{-} j}^{\beta}$

$\delta W$

$\delta W^{p}$

$\delta W_{d}$
Sphere radii

Sample volume at time-step $\beta$

Translational speed of particle $i$

Total boundary work input between time-steps 0 and $\beta-1$ or $\beta$

Total distortional work between time-steps 0 and $\beta-1$ or $\beta$

Total volumetric work between time-steps 0 and $\beta-1$ or $\beta$

Reference Cartesian axes

Coordination number

Mechanical coordination number

Interparticle overlap

Identifier of one time-step during shearing

Friction dissipated at contact $j$ during time-step $\beta$

Increment of tangential component of strain energy at contact $j$ during time-step $\beta$

Increment of boundary work input per unit volume

Increment of total input energy available for dissipation

Increment of distortional work per unit volume 
Increment of volumetric work per unit volume

$\delta \boldsymbol{\alpha}_{t} \quad$ Increment of relative shear displacement

$\delta \varepsilon_{q} \quad$ Increment of triaxial shear strain

$\delta \varepsilon_{q}^{e}, \delta \varepsilon_{q}^{p} \quad$ Elastic/plastic increments of triaxial shear strain

$\delta \varepsilon_{v} \quad$ Increment of volumetric strain

$\delta \varepsilon_{v}^{e}, \delta \varepsilon_{v}^{p} \quad$ Elastic/plastic increments of volumetric strain

$\delta \varepsilon_{x}, \delta \varepsilon_{y}, \delta \varepsilon_{z} \quad$ Incremental normal strains

$\delta \Phi$

Dissipative part of the plastic work

$\Delta E$

Error term in the energy balance

$\varepsilon_{q}$

Deviatoric strain

$\varepsilon_{v} \quad$ Volumetric strain

$\varepsilon_{x}, \varepsilon_{y}, \varepsilon_{z} \quad$ Normal strains

$\dot{\varepsilon}_{z} \quad$ Normal strain rate during triaxial compression

$\eta$

Stress ratio $q / p^{\prime}$

$\kappa$

Negative slope of the unloading-reloading line in $(1+e): \ln \left(p^{\prime}\right)$ space

$\mu$

Interparticle friction coefficient

$v$

Sample Poisson's ratio

$v_{p} \quad$ Particle Poisson's ratio

$\sigma_{x}^{\prime}, \sigma_{y}^{\prime}, \sigma_{z}^{\prime} \quad$ Effective normal stresses for the sample 


$\begin{array}{ll}\tau_{x y}, \tau_{x z}, \tau_{y z} & \text { Effective shear stresses for the sample } \\ \Phi_{p q} & \text { Second-order fabric tensor } \\ \psi_{0} & \text { Initial state parameter after isotropic compression } \\ \omega_{i} & \text { Rotational speed of particle } i\end{array}$

\section{References}

Been, K. \& Jefferies, M. G. (1985). A state parameter for sands. Géotechnique 35, No. 2, 99-112.

Belytschko, T., Liu, W. K., Moran, B. \& Elkhodary, K. I. (2013). Nonlinear finite elements for continua and structures, 2nd edn. New York, USA: Wiley.

Bernhardt, M. L., Biscontin, G. \& O'Sullivan, C. (2016). Experimental validation study of 3D direct simple shear DEM simulations. Soils Found. 56, No. 3, 336-347.

Berrill, J. B. \& Davis, R. O. (1985). Energy dissipation and seismic liquefaction of sands: revised model. Soils Found. 25, No. 2, 106-118.

Bi, Z., Sun, Q., Jin, F. \& Zhang, M. (2011). Numerical study on energy transformation in granular matter under biaxial compression. Granul. Matter 13, No. 4, 503-510.

Bolton, M. D., Nakata, Y. \& Cheng, Y. P. (2008). Micro- and macro-mechanical behaviour of DEM crushable materials. Géotechnique 58, No. 6, 471-480.

Cheng, Y. P., Bolton, M. D. \& Nakata, Y. (2004). Crushing and plastic deformation of soils simulated using DEM. Géotechnique 54, No. 2, 131-141. 
Ciantia, M. O., Arroyo, M., Calvetti, F. \& Gens, A. (2015). An approach to enhance efficiency of DEM modelling of soils with crushable grains. Géotechnique 65, No. 2, 91-110.

Collins, I. F. \& Hilder, T. (2002). A theoretical framework for constructing elastic/plastic constitutive models of triaxial tests. Int. J. Num. Anal. Meth. Geomech. 26, No. 13, 1313-1347.

Collins, I. F. \& Muhunthan, B. (2003). On the relationship between stress-dilatancy, anisotropy, and plastic dissipation for granular materials. Géotechnique 53, No. 7, 611-618.

Cundall, P. (1988). Computer simulations of dense sphere assemblies. In Micromechanics of Granular Materials (eds M. Satake and J. Jenkins), pp. 113-123. Amsterdam: Elsevier.

Cundall, P. A. \& Strack, O. D. L. (1979). A discrete numerical model for granular assemblies. Géotechnique 29, No. 1, 47-65.

da Cruz F., Emam, S., Prochnow, M., Roux, J.-N. \& Chevoir, F. (2005). Rheophysics of dense granular materials: Discrete simulation of plane shear flows. Phys. Rev. E 72, 021309.

Einav, I. (2007). Breakage mechanics-Part II: Modelling granular materials. J. Mech. Phys. Solids 55, No. 6, 1298-1320.

El Shamy, U. \& Denissen, C. (2012). Microscale energy dissipation mechanisms in cyclically-loaded granular soils. Geotech. Geol. Eng. 30, No. 2, 343-361.

Figueroa, J. L., Saada, A. S., Liang, L. \& Dahisaria, N. M. (1994). Evaluation of soil liquefaction by energy principles. J. Geotech. Eng. 120, No. 9, 1554-1569.

Gajo, A. \& Muir Wood, D. (1999). Severn-Trent sand: a kinematic-hardening constitutive model: the q-p formulation. Géotechnique 49, No. 5, 595-614. 
Gens, A. \& Potts, D. M. (1988). Critical state models in computational geomechanics. Eng. Comput. 5, No. 3, 178-197.

Guo, N. \& Zhao, J. (2013). The signature of shear-induced anisotropy in granular media. Comput. Geotech. 47, 1-15.

Hanley, K. J., Huang, X., O'Sullivan, C. \& Kwok, F. C. Y. (2014). Temporal variation of contact networks in granular materials. Granul. Matter 16, No. 1, 41-54.

Hanley, K. J., O'Sullivan, C. \& Huang, X. (2015). Particle-scale mechanics of sand crushing in compression and shearing using DEM. Soils Found. 55, No. 5, 1100-1112.

Houlsby, G. T. (1981). A study of plasticity theories and their applicability to soils. PhD thesis, University of Cambridge.

Huang, X., Hanley, K. J., O'Sullivan, C. \& Kwok, F. C. Y. (2014a). Effect of sample size on the response of DEM samples with a realistic grading. Particuology 15, 107-115.

Huang, X., Hanley, K. J., O'Sullivan, C. \& Kwok, C. Y. (2014b). Exploring the influence of interparticle friction on critical state behaviour using DEM. Int. J. Numer. Anal. Met. 38, No. 12, $1276-1297$.

Huang, X., Hanley, K. J., O’Sullivan, C. \& Kwok, C.-Y. (2017). Implementation of rotational resistance models: a critical appraisal. Particuology, doi:10.1016/j.partic.2016.08.007.

Itasca Consulting Group (2008). PFC3D: Particle flow code in three dimensions User's Guide, 4th edn. Minneapolis: ICG.

Jefferies, M. G. (1993). Nor-Sand: a simple critical state model for sand. Géotechnique 43, No. 1, 91103. 
Jefferies, M. G. \& Been, K. (2006). Soil liquefaction: a critical state approach. London, UK: Taylor and Francis.

Jefferies, M. G. \& Shuttle, D. A. (2002). Dilatancy in general Cambridge-type models. Géotechnique 52, No. 9, 625-638.

Kamai, R. \& Boulanger, R. W. (2012). Single-element simulations of partial-drainage effects under monotonic and cyclic loading. Soil Dyn. Earthq. Eng. 35, 29-40.

Kikumoto, M., Muir Wood, D. \& Russell, A. (2010). Particle crushing and deformation behaviour. Soils Found. 50, No. 4, 547-563.

Law, K. T., Cao, Y. L., \& He, G. N. (1990). An energy approach for assessing seismic liquefaction potential. Can. Geotech. J. 27, No. 3, 320-329.

Manzari, M. T. \& Dafalias, Y. F. (1997). A critical state two-surface plasticity model for sands. Géotechnique 47, No. 2, 255-272.

McDowell, G. R. \& Bolton, M. D. (1998). On the micro-mechanics of crushable aggregates. Géotechnique 48, No. 5, 667-679.

Muir Wood, D. (1990). Soil behaviour and critical state soil mechanics. Cambridge, UK: Cambridge University Press.

$\mathrm{Ng}, \mathrm{T}$. (2009). Discrete element method simulations of the critical state of a granular material. Int. J. Geomech. 9, No. 5, 209-216.

O’Sullivan, C. (2014). Advancing geomechanics using DEM. Proc. International Symposium on Geomechanics from Micro to Macro. 1, 21-32. 
O'Sullivan, C. \& Bray, J. D. (2004). Selecting a suitable time step for discrete element simulations that use the central difference time integration scheme. Eng. Comput. 21, No. 2/3/4, 278-303.

Plimpton, S. (1995). Fast parallel algorithms for short-range molecular dynamics. J. Comput. Phys. 117, 1-19.

Potyondy, D. O. \& Cundall, P. A. (2004). A bonded-particle model for rock. Int. J. Rock Mech. Min. 41, No. 8, 1329-1364.

Roscoe, K. H. \& Burland, J. B. (1968). On the generalized stress-strain behaviour of 'wet clay'. In Engineering plasticity (eds J. Heyman and F. A. Leckie), pp. 535-609. Cambridge, UK: Cambridge University Press.

Roscoe, K. H. \& Schofield, A. N. (1963). Mechanical behaviour of an idealized wet clay. Proc. European Conf. on Soil Mechanics and Foundation Eng. 1, 47-54.

Satake, M. (1982). Fabric tensor in granular materials. In Proceedings of the IUTAM symposium on deformations and failure of granular materials 1982 (eds P. A. Vermeer and H. J. Luger), pp. 63-68. Rotterdam, the Netherlands: Balkema.

Schofield, A. \& Wroth, P. (1968). Critical state soil mechanics. London, UK: McGraw-Hill.

Seed, H. B., Wong, R. T., Idriss, I. M. \& Tokimatsu, K. (1986). Moduli and damping factors for dynamic analyses of cohesionless soils. J. Geotech. Eng. 112, No. 11, 1016-1032.

Sitharam, T. G. \& Vinod, J. S. (2010). Evaluation of shear modulus and damping ratio of granular materials using discrete element approach. Geotech. Geol. Eng. 28, No. 5, 591-601.

Sun, Q., Song, S., Jin, F. \& Bi, Z. (2011). Elastic energy and relaxation in triaxial compressions. Granul. Matter 13, No. 6, 743-750. 
Taylor, D. W. (1948). Fundamentals of soil mechanics. New York, USA: Wiley.

Thornton, C. (2000). Numerical simulations of deviatoric shear deformation of granular media. Géotechnique 50, No. 1, 43-53.

Trifunac, M. D. (1995). Empirical criteria for liquefaction in sands via standard penetration tests and seismic wave energy. Soil Dyn. Earthq. Eng. 14, 419-426.

Vucetic, M. \& Dobry, R. (1991). Effect of soil plasticity on cyclic response. J. Geotech. Eng. 117, No. 1, 89-107.

Wang, J. \& Yan, Y. (2012). DEM analysis of energy dissipation in crushable soils. Soils Found. 52, No. 4, 644-657.

Wensrich, C. M. \& Katterfeld, A. (2012). Rolling friction as a technique for modelling particle shape in DEM. Powder Technol. 217, 409-417.

Witt, W., Köhler, U. \& List, J. (2004). Direct imaging of very fast particles opens the application of the powerful (dry) dispersion for size and shape characterization. Proc. PARTEC 2004, Nürnberg (CD proceedings).

Yan, W. M. \& Dong, J. (2011). Effect of particle grading on the response of an idealized granular assemblage. Int. J. Geomech. 11, No. 4, 276-285.

Zamani, N. \& El Shamy, U. (2013). Discrete-element method simulations of the response of soilfoundation-structure systems to multidirectional seismic motion. Int. J. Geomech. 13, No. 5, 595-610.

Zhang, W., Wang, J. \& Jiang, M. (2013). DEM-aided discovery of the relationship between energy dissipation and shear band formation considering the effects of particle rolling resistance. J. Geotech. Geoenviron. 139, No. 9, 1512-1527. 
Zhou, B., Huang, R. \& Wang, J. (2013). Evolution of sand crushability and its effect on particle-scale energy allocation. AIP Conference Proc. (Powders and Grains 2013, Sydney, Australia). 1542, 923926.

Zytynski, M., Randolph, M. F., Nova, R. \& Wroth, C. P. (1978). On modelling the unloadingreloading behaviour of soils. Int. J. Num. Anal. Meth. Geomech. 2, No. 1, 87-93. 


\section{Figures}

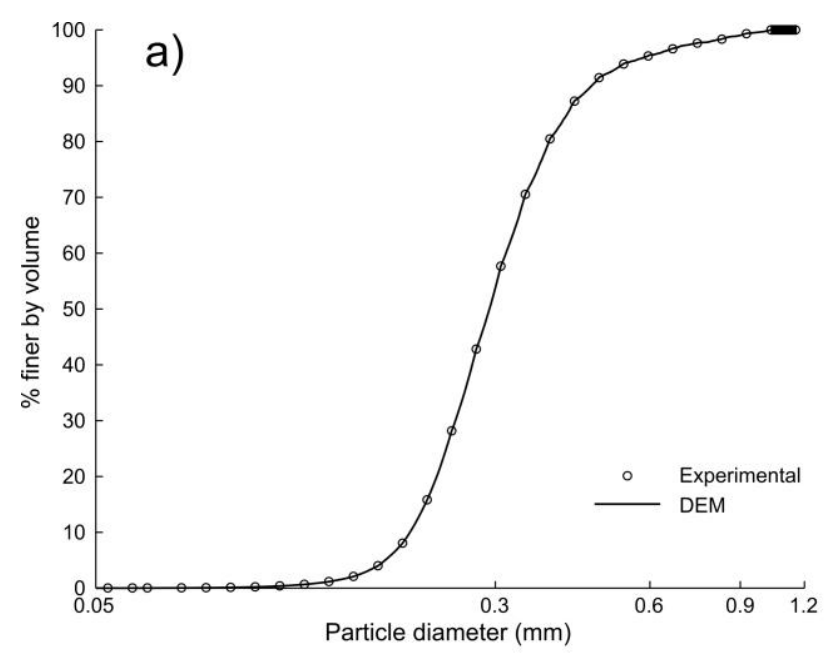

b)

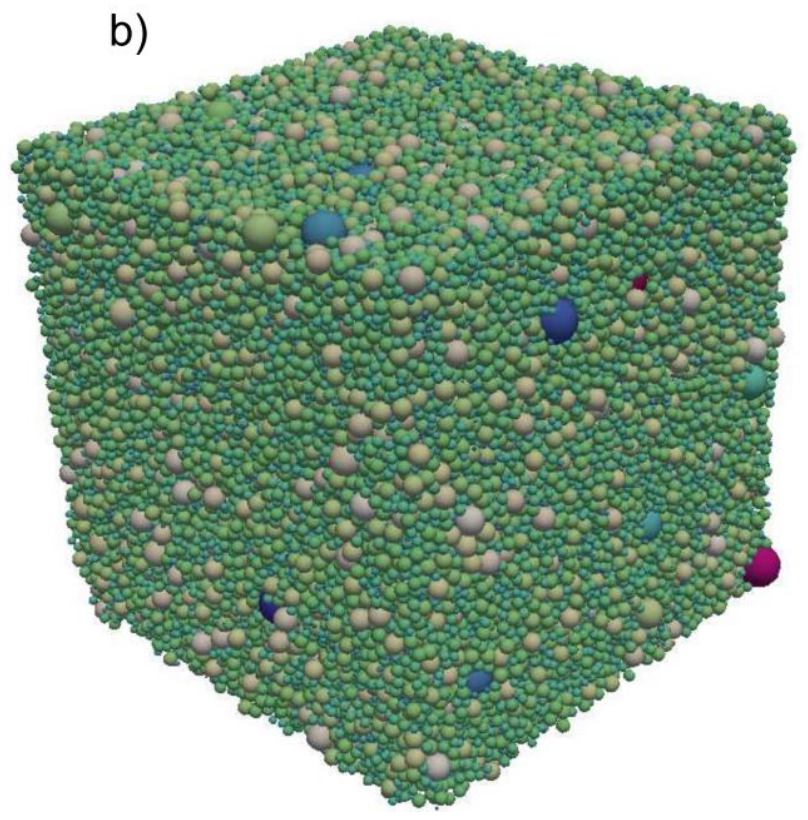

Fig. 1. a) Particle size distribution by volume of the numerical grading used for this study compared with the size distribution for Dunkirk sand measured by sieving; b) the densest numerical sample after isotropic compression to $500 \mathrm{kPa}$ 

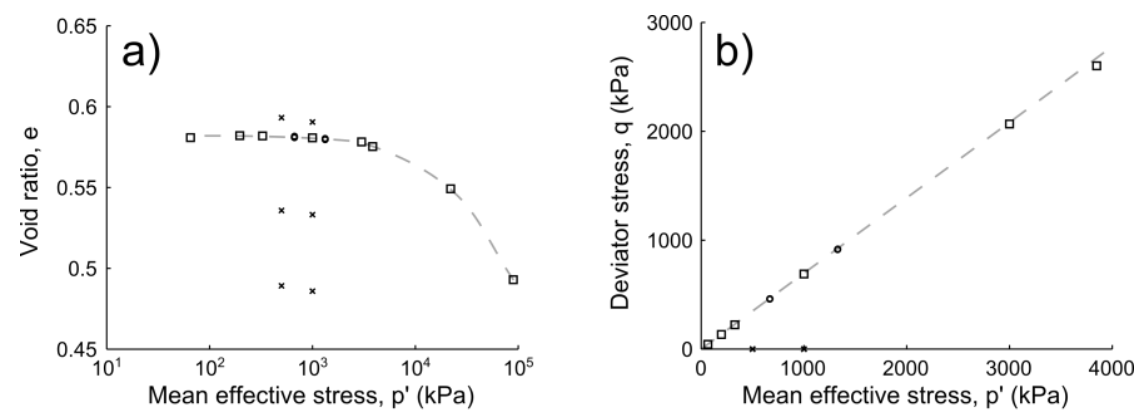

- 101,623 particles

- 43,906 particles

End of isotropic compression

Fitted CSL

Fig. 2. Critical state line in a) $\boldsymbol{e}-\log \left(\boldsymbol{p}^{\prime}\right)$ and b) $\boldsymbol{q}-\boldsymbol{p}^{\prime}$ spaces
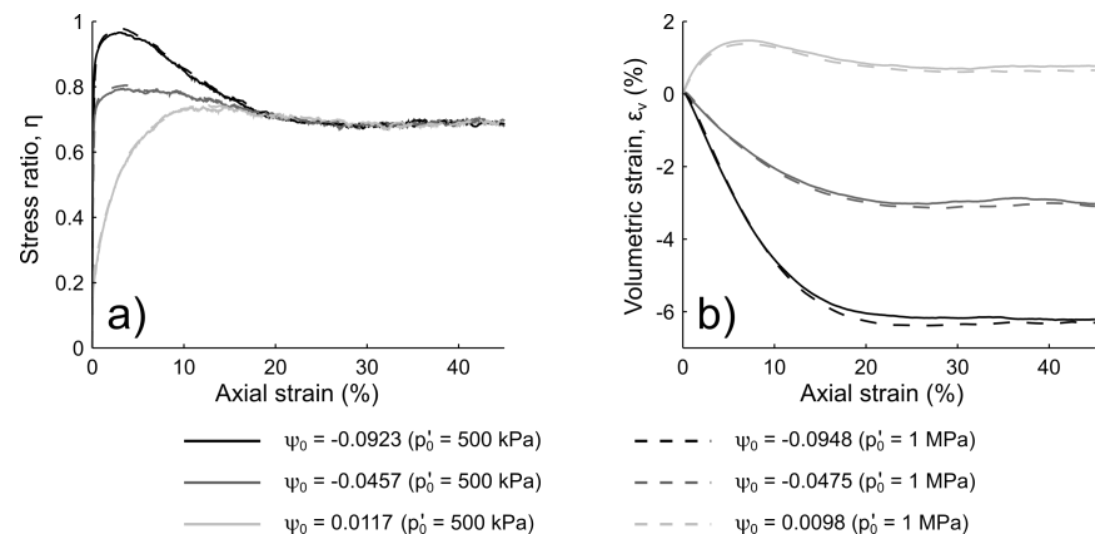

Fig. 3. Stress ratio, $\eta$, and percentage volumetric strain, $\varepsilon_{v}$, against axial strain (\%) for all six triaxial simulations 

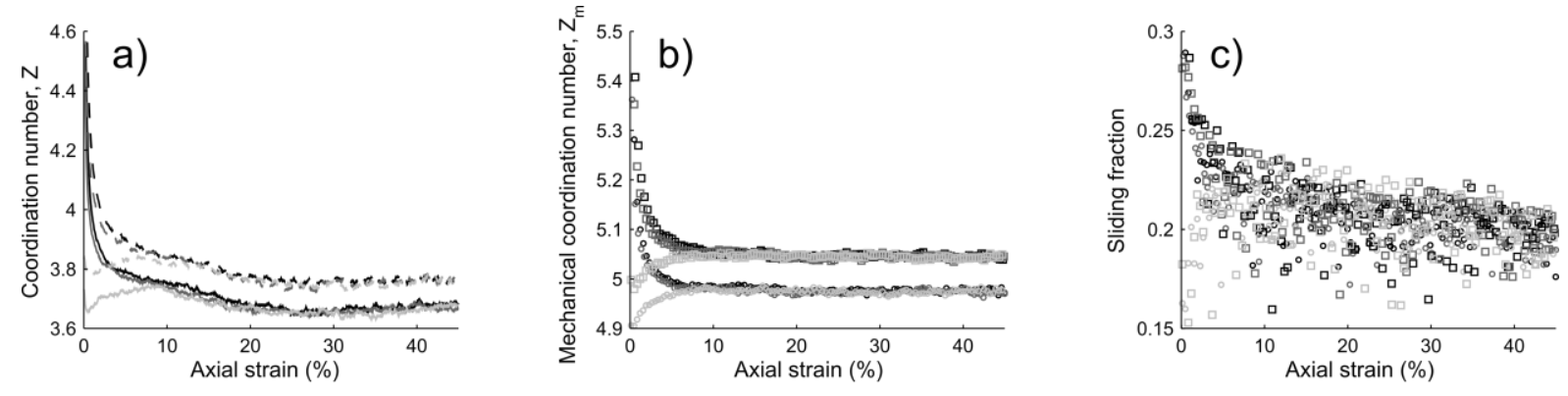

\begin{tabular}{lll}
\hline & $\circ$ & $\psi_{0}=-0.0923\left(\mathrm{p}_{0}^{\prime}=500 \mathrm{kPa}\right)$ \\
\hline$\ldots \ldots$ & $\square$ & $\psi_{0}=-0.0948\left(\mathrm{p}_{0}^{\prime}=1 \mathrm{MPa}\right)$
\end{tabular}

-1 ¿ $\psi_{0}=-0.0457\left(\mathrm{p}_{0}^{\prime}=500 \mathrm{kPa}\right)$

$\psi_{0}=0.0117\left(p_{0}^{\prime}=500 \mathrm{kPa}\right)$

- - ⿱ 口 $\quad \psi_{0}=-0.0475\left(\mathrm{p}_{0}^{\prime}=1 \mathrm{MPa}\right)$

$\psi_{0}=0.0098\left(\mathrm{p}_{0}^{\prime}=1 \mathrm{MPa}\right)$

Fig. 4. Coordination number $(\mathrm{Z})$, mechanical coordination number $\left(\mathrm{Z}_{\mathrm{m}}\right)$ and sliding contact fraction against axial strain (\%)

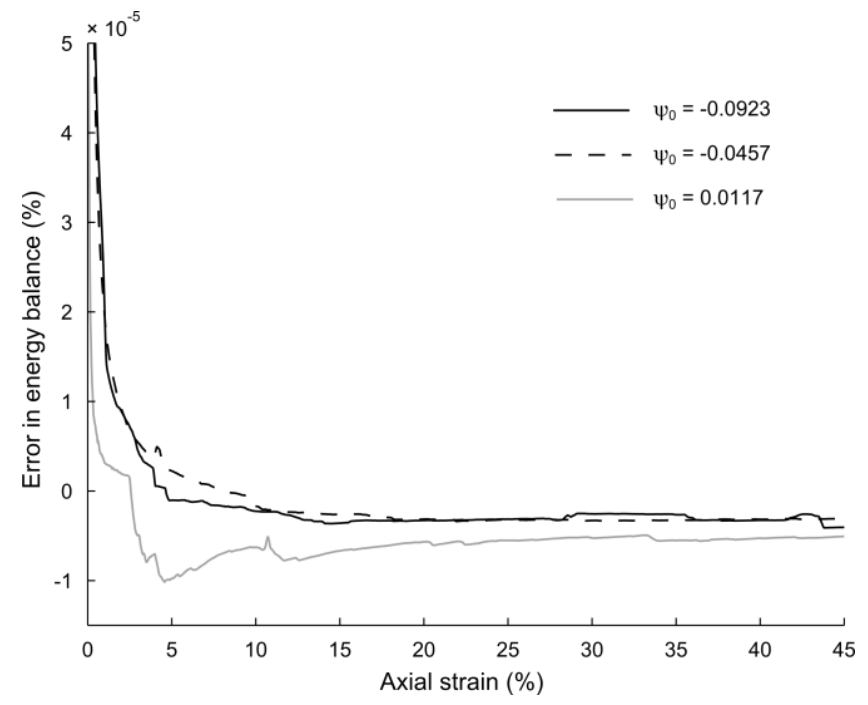

Fig. 5. Error in the energy balance expressed as a percentage of the boundary work against axial strain

(\%) for those three simulations with $\mathrm{p}_{0}{ }^{\prime}=500 \mathrm{kPa}$ 

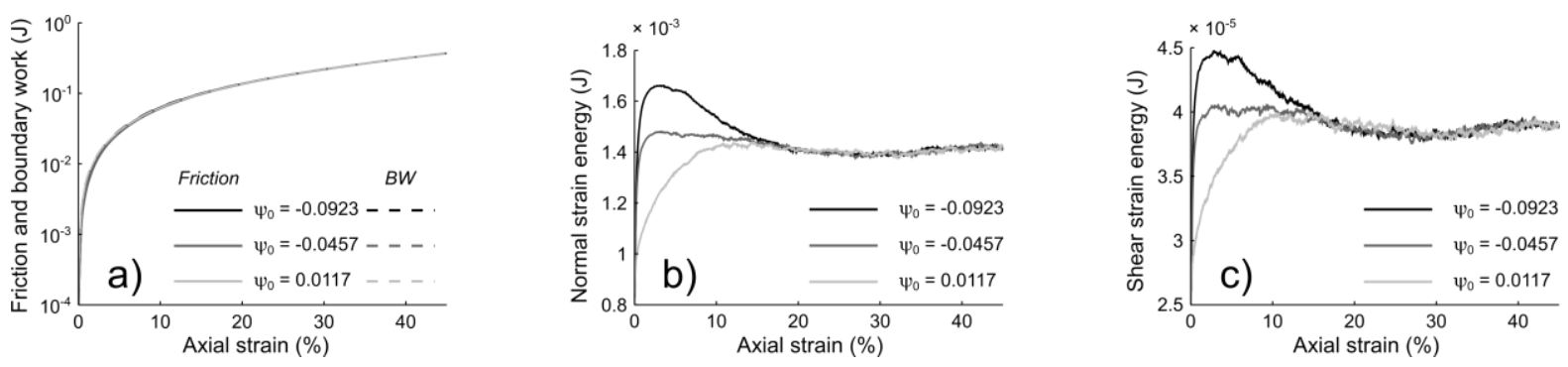

Fig. 6. The evolution of four energy terms (J) against axial strain (\%): a) energy dissipated by frictional sliding and boundary work; b) normal component of strain energy; c) shear component of strain energy
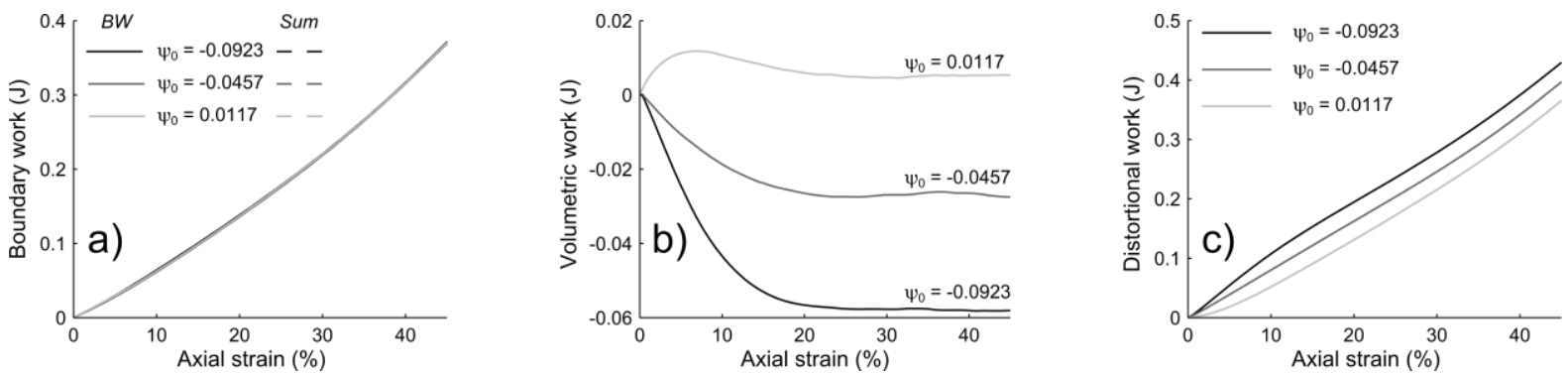

Fig. 7. The evolution of boundary work, volumetric work, distortional work and the sum of volumetric and distortional work, all in $\mathrm{J}$, against axial strain (\%) 


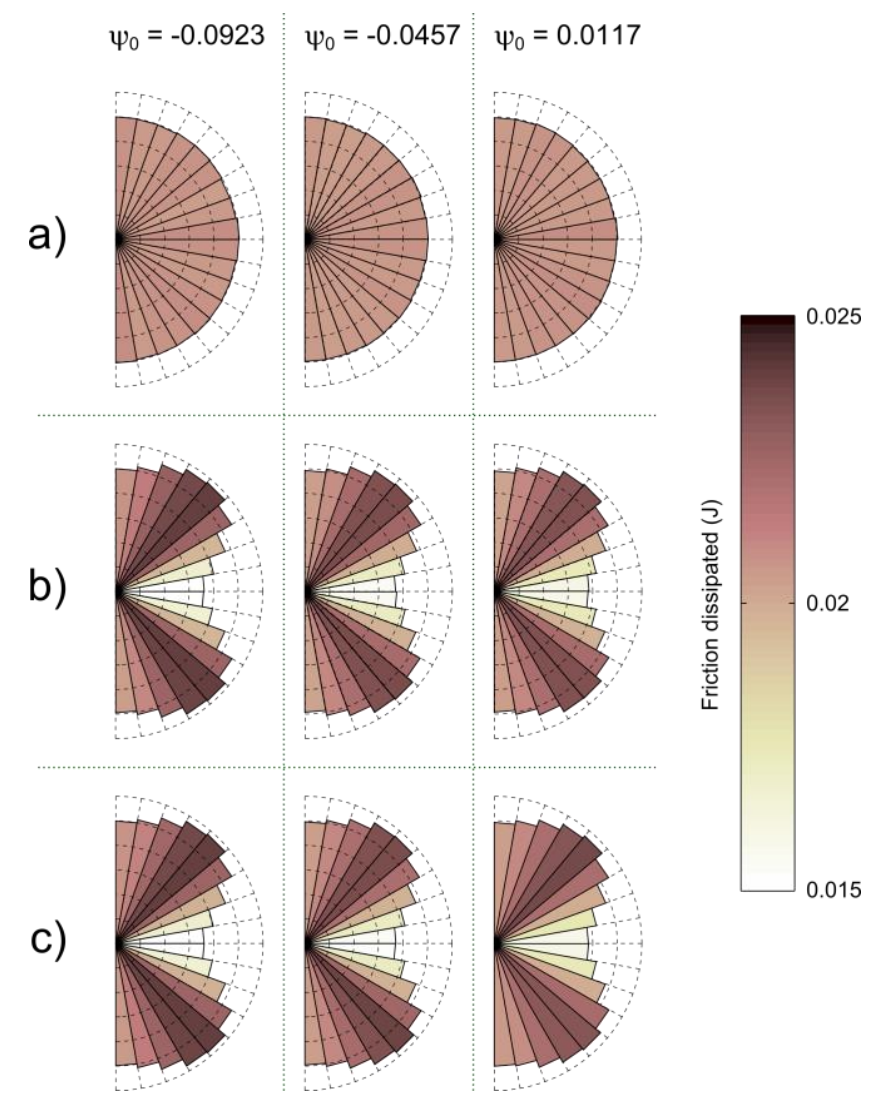

Fig. 8. Rose diagrams showing the total frictional dissipation $(\mathrm{J})$ at critical state at each orientation. Rows a), b) and c) show projections onto the $x-y, x-z$ and $y-z$ planes, respectively. The lengths and colours of each segment indicate the total frictional dissipation for contacts at that orientation 


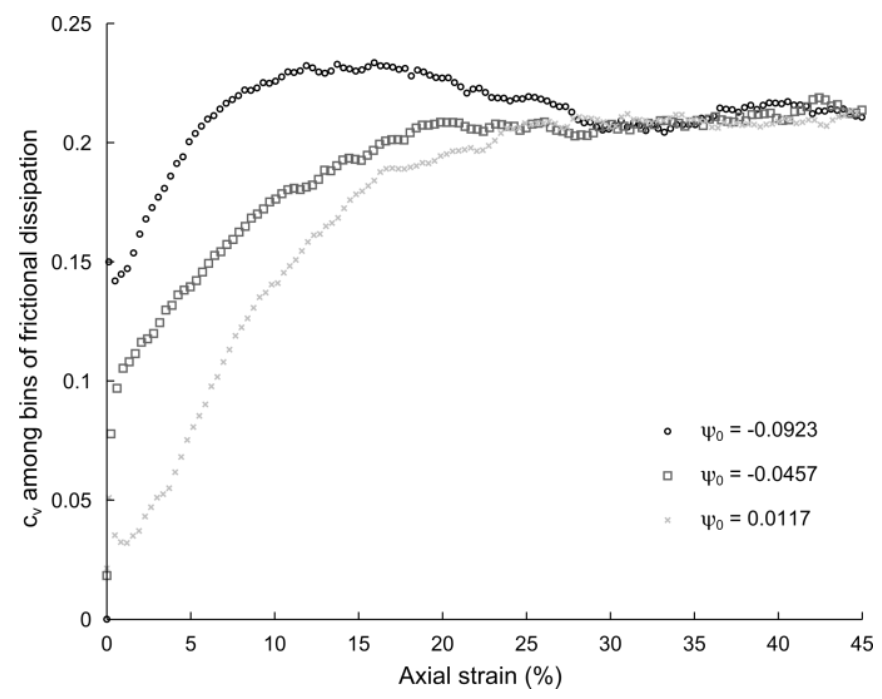

Fig. 9. Coefficient of variation among the 36 bins for total frictional dissipation for projections onto the $x-z$ and $y-z$ planes against axial strain (\%) 


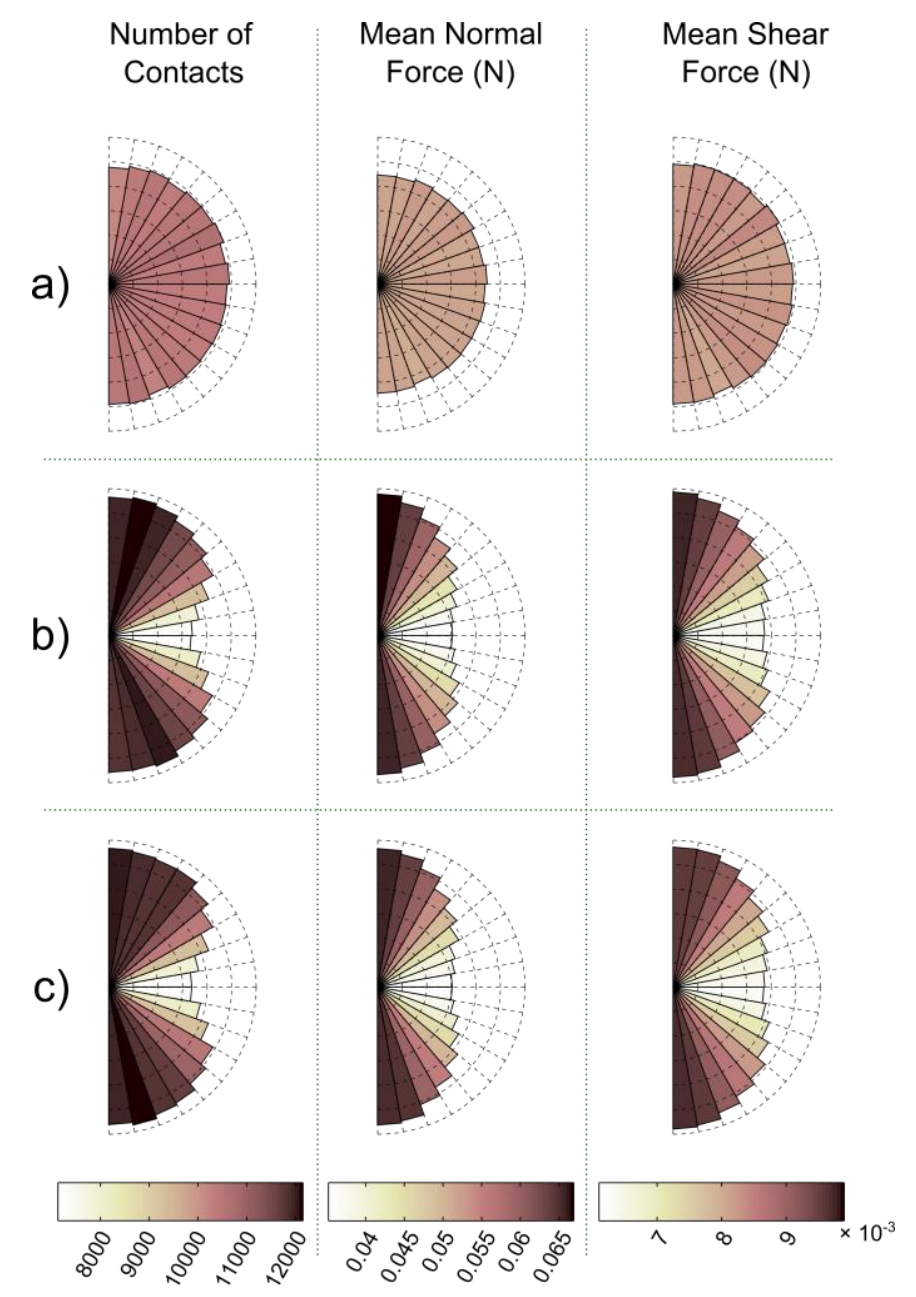

Fig. 10. Rose diagrams showing the number of interparticle contacts and the mean normal and shear forces $(\mathrm{N})$ for one simulation with $\psi_{0}=-0.0457$. As in Fig. 8, rows a), b) and c) show projections onto the $x-y, x-z$ and $y-z$ planes, respectively 


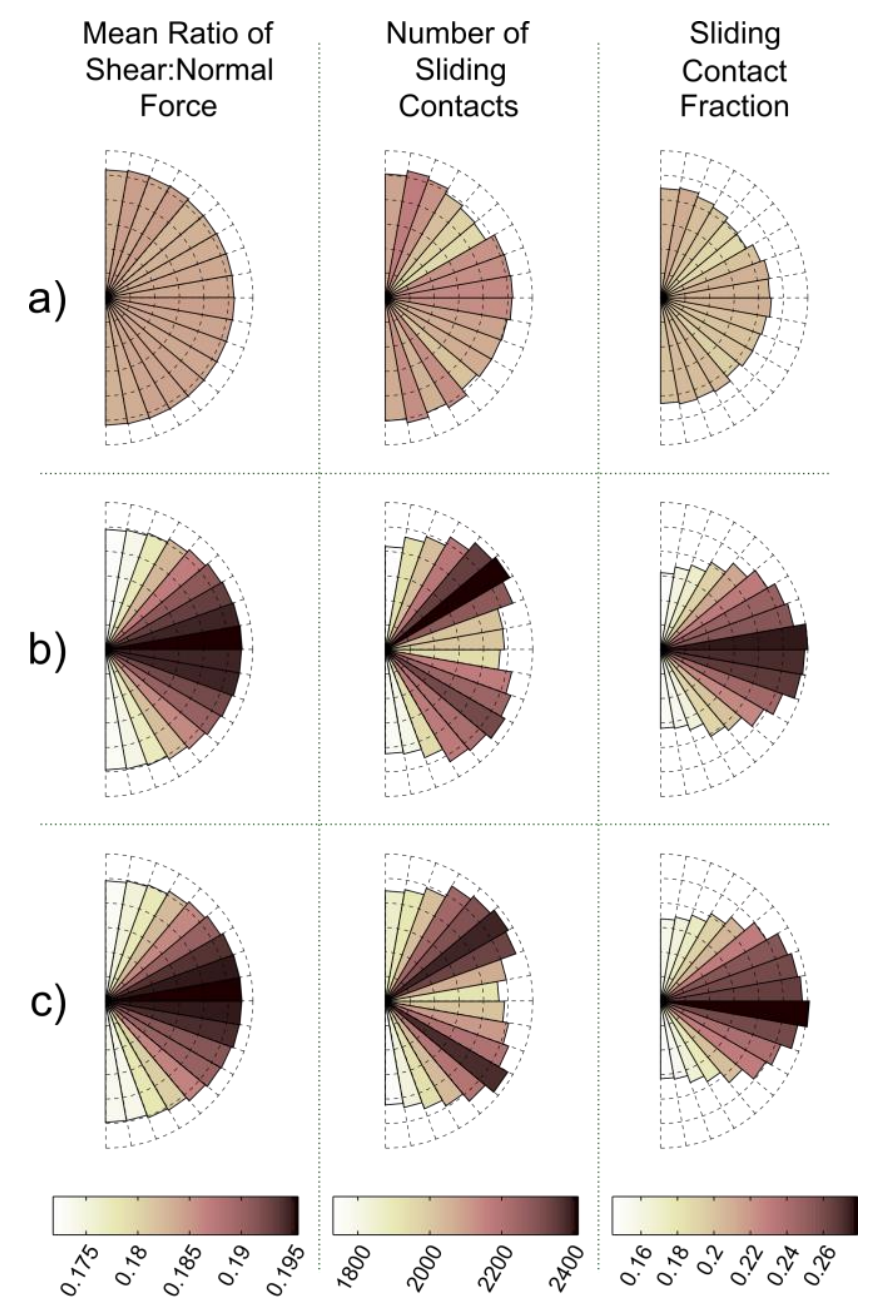

Fig. 11. Rose diagrams showing the mean ratio of shear to normal force, the number of sliding contacts and the fraction of sliding contacts at critical state for $\psi_{0}=-0.0457$. Rows a), b) and c) respectively show projections onto the $x-y, x-z$ and $y-z$ planes 


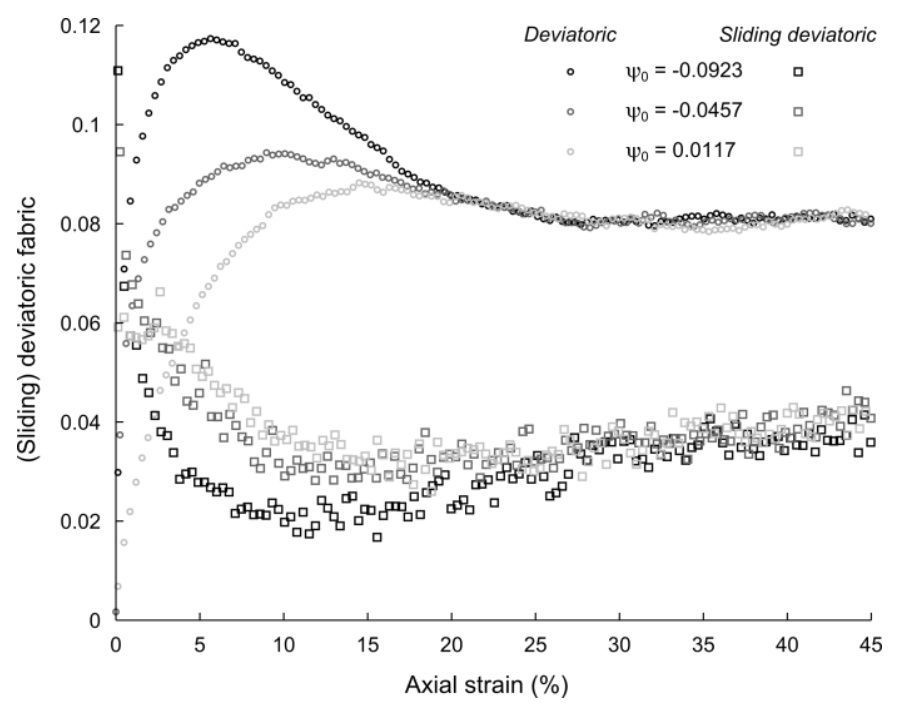

Fig. 12. Deviatoric fabric of all contacts (o) and sliding contacts only (口) against axial strain (\%)

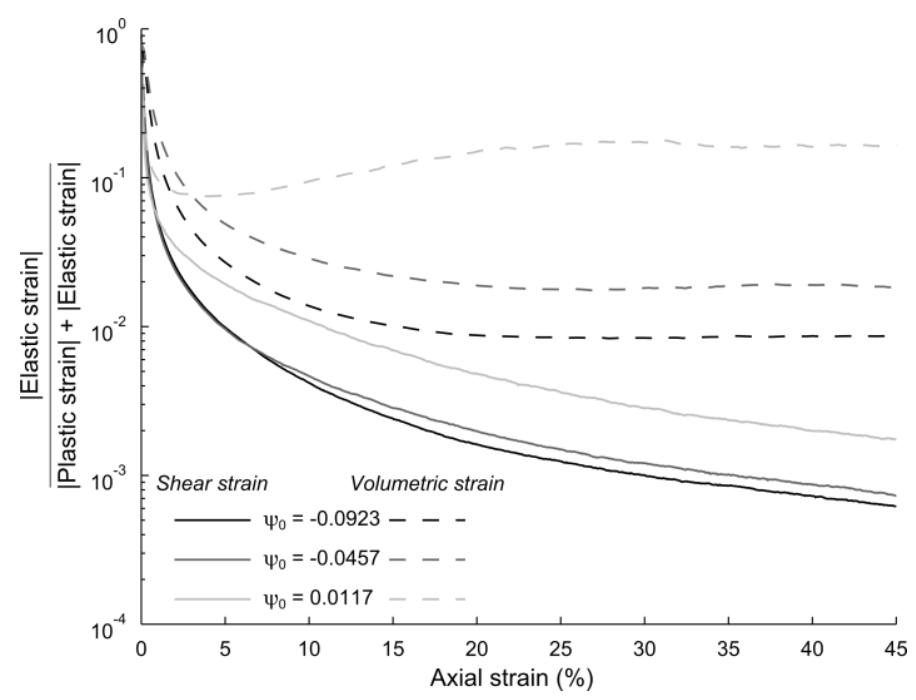

Fig. 13. The elastic proportions of the total shear and volumetric strains against axial strain (\%) 

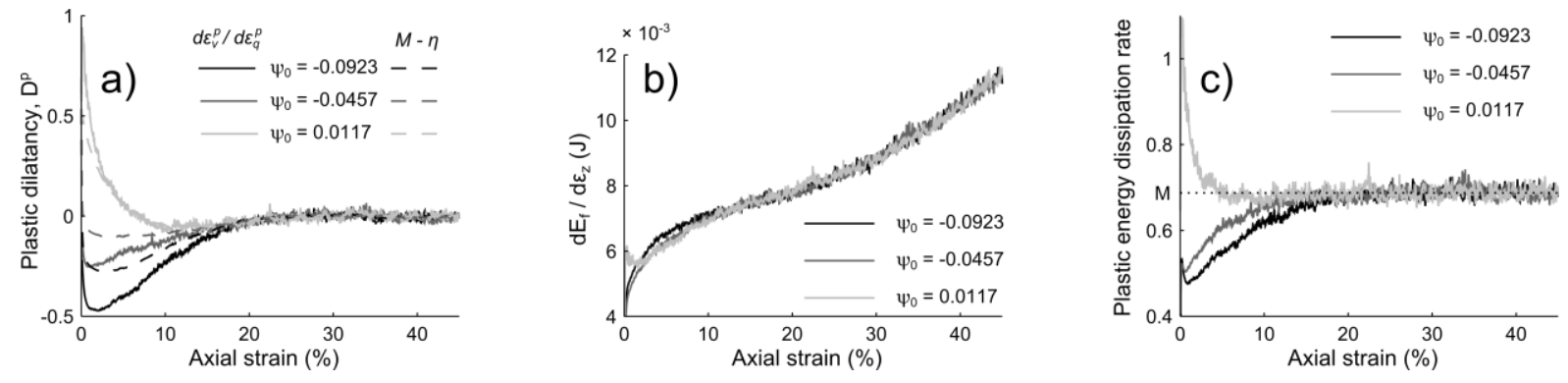

Fig. 14. a) Dilation rate computed using plastic strains, $D^{\mathrm{p}}$; b) rate of change of frictional dissipation with respect to axial strain (J); c) dimensionless plastic energy dissipation rate, all plotted against axial strain $(\%)$
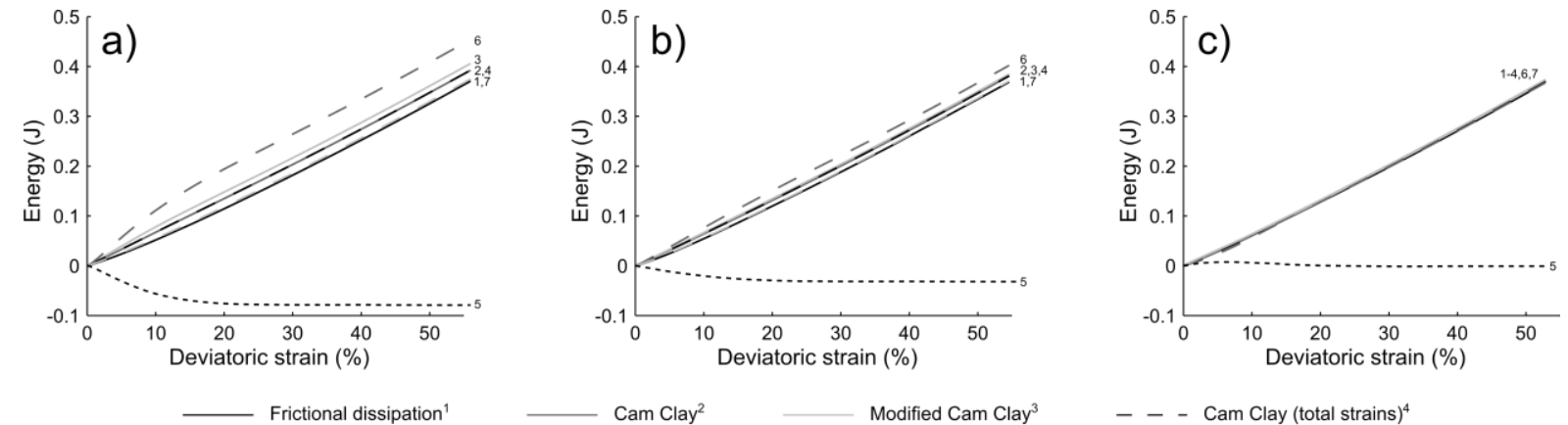

$\begin{aligned} \text { Frictional dissipation }^{1} & \\ \ldots \ldots \ldots \ldots . . . & \text { Equation }(23)^{5}\end{aligned}$

$\mathrm{Cam} \mathrm{Clay}^{2} \quad$ Modified Cam Clay $^{3}$

Equation $(24)^{6}$

- - - Cam Clay (total strains)

Equation $(25)^{7}$

Fig. 15. Comparison of work equations and frictional dissipation, all in $\mathrm{J}$, plotted against deviatoric strain (\%) for $\psi_{0}$ of a) -0.0923, b) -0.0457 , and c) 0.0117 . The superscript numerals in the legend indicate the corresponding lines on the figure 

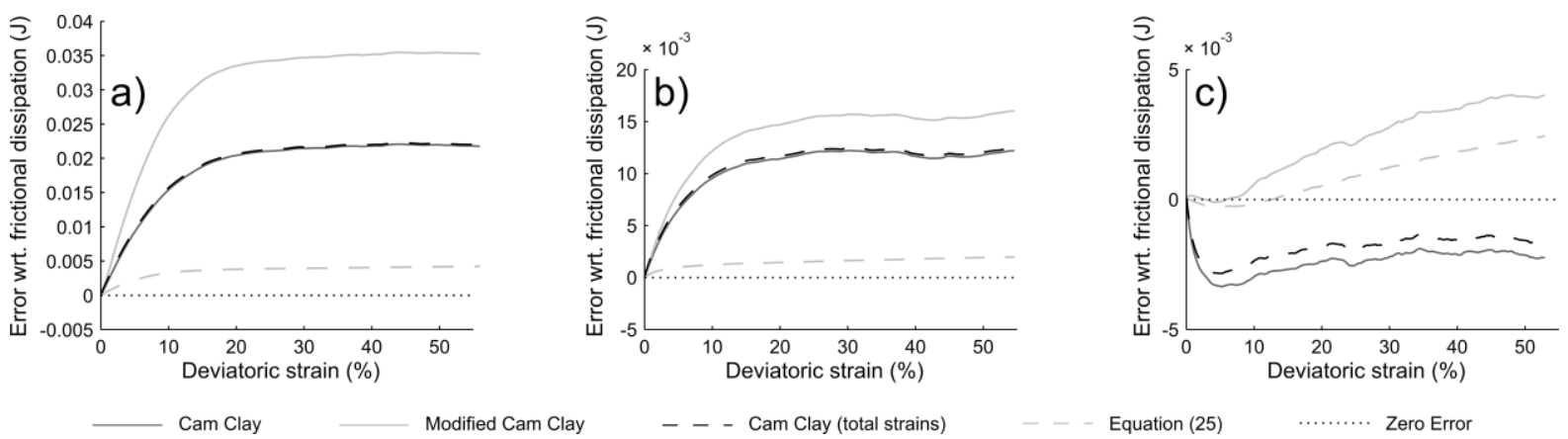

- - - Cam Clay (total strains)

Equation (25)

Zero Error

Fig. 16. Difference between the work equations and frictional dissipation $(\mathrm{J})$ against deviatoric strain

(\%) for $\psi_{0}$ of a) -0.0923, b) -0.0457 , and c) 0.0117 . A positive difference denotes an over-prediction of dissipation 